ARTICLE

https://doi.org/10.1038/s41467-020-19477-3

\title{
The regulatory function of the AAA4 ATPase domain of cytoplasmic dynein
}

\author{
Xinglei Liu (1) ${ }^{1}$, Lu Rao $^{1} \&$ Arne Gennerich ${ }^{1 凶}$
}

Cytoplasmic dynein is the primary motor for microtubule minus-end-directed transport and is indispensable to eukaryotic cells. Although each motor domain of dynein contains three active AAA+ ATPases (AAA1, 3, and 4), only the functions of AAA1 and 3 are known. Here, we use single-molecule fluorescence and optical tweezers studies to elucidate the role of AAA4 in dynein's mechanochemical cycle. We demonstrate that AAA4 controls the priming stroke of the motion-generating linker, which connects the dimerizing tail of the motor to the AAA+ ring. Before ATP binds to AAA4, dynein remains incapable of generating motion. However, when AAA4 is bound to ATP, the gating of AAA1 by AAA3 prevails and dynein motion can occur. Thus, AAA1, 3, and 4 work together to regulate dynein function. Our work elucidates an essential role for AAA4 in dynein's stepping cycle and underscores the complexity and crosstalk among the motor's multiple AAA+ domains.

\footnotetext{
${ }^{1}$ Department of Anatomy and Structural Biology and Gruss Lipper Biophotonics Center, Albert Einstein College of Medicine, Bronx, NY 10461, USA.

凶email: arne.gennerich@einsteinmed.org
} 
T he cytoplasmic dynein motor complex (referred to here as dynein) is an intricate and ubiquitous biological nanomachine that is responsible for a vast array of functions including intracellular transport of organelles and signaling complexes, nuclear transport during neuronal migration, regulation of mitotic spindle length and positioning, and removal of checkpoint proteins from the kinetochore during cell division (reviewed in refs. ${ }^{1-4}$ ). Thus, it is not surprising that dynein dysfunction has been linked to a growing number of human diseases termed "dyneinopathies" 5,6 including malformation of cortical development $(\mathrm{MCD})^{7-9}$, spinal muscular atrophy (SMA) $)^{10,11}$, SMA with lower extremity predominance (SMALED $^{12,13}$, and others ${ }^{14-16}$. However, dynein's molecular mechanism, the regulatory functions of its subunits, and the molecular effects of disease mutations, remain largely unclear.

Dynein is the largest $(\sim 1.4 \mathrm{MDa})$ and most complex cytoskeletal motor protein. It is comprised of two identical heavy chains (HCs) and several subunits ${ }^{4}$. The dynein $\mathrm{HC}$ contains an N-terminal dimerizing tail domain and a C-terminal motor domain (MD) or "head" with six tandem-linked AAA+ ATPase modules (AAA: ATPase associated with various cellular activities) arranged in a ring (AAA1-6) (Fig. 1a). Only AAA1, 3, and 4 hydrolyze ATP ${ }^{1}$. Three elongated structures emerge from the AAA ring: A 15-nm coiledcoil "stalk" that protrudes from AAA4 and separates dynein's MTbinding domain (MTBD) from the AAA+ ring ${ }^{17,18}$, an antiparallel coiled-coil called the buttress ${ }^{17}$ (or strut ${ }^{18}$ ) that emerges from AAA5 and contacts the stalk, and a $~ 10$-nm "linker" that extends from AAA1 and connects the AAA+ ring to the tail ${ }^{19-21}$. The linker undergoes conformational changes $19,22,23$ that generate unidirectional motion and force, and it also controls the buttressmediated sliding of the stalk helices to shift between weak and strong MT-binding states ${ }^{24}$ (Fig. 1a, b).

Dynein can move processively (the ability to take multiple steps before dissociating ${ }^{25}$ ), and we are only beginning to understand how dynein generates continuous unidirectional motion under opposing forces. In mammalian dynein, activation by its largest cofactor, dynactin, together with a cargo adapter (e.g., BICD2) is required to convert dynein from a diffusive ${ }^{26 / \text { weakly processive }}{ }^{27}$ motor to an ultraprocessive motor ${ }^{28,29}$. This activation involves realignment of the MDs into a parallel orientation where both $\mathrm{MDs}$ can readily bind the $\mathrm{MT}^{30-33}$. Once activated, processive movement can occur under load ${ }^{34}$, with one head binding the MT tightly while the other detaches and advances. Recent studies showed the cargo adapter-dependent recruitment of two dynein motors arranged side-by-side on a MT (i.e., four MDs in parallel $)^{35,36}$. Recruitment of a second motor likely increases processivity by reducing the dissociation rate of the entire complex from the MT. While these studies provide important insights into the mechanisms that facilitate dynein processivity, a fundamental question remains: how do the convoluted interactions of dynein's multiple AAA+ domains control the shifts between strong and weak MT-binding states of dynein's MTBDs so that the forcebearing leading head stays bound to the MT while the partner head detaches and advances?

This question has been partially answered by studies that demonstrate that the stalk is a bidirectional communication pathway between the AAA+ ring and MTBD $24,37,38$. The nucleotide states of the MD affect MT binding and vice versa ${ }^{39-41}$. For example, it is known that ATP binding to AAA1 and ADP binding to AAA3 both reduce MT-binding strength ${ }^{42}$. Conversely, ADP binding to AAA1 has the opposite effect ${ }^{42}$. In addition, $\mathrm{we}^{42}$ and others ${ }^{43}$ reported that AAA3 acts as a "gate" for ATP-induced MT release. ATP binding to AAA1 induces MT release only if AAA3 is in the post-hydrolysis state. When force is applied to the linker, ATP binding to AAA3 "opens" the gate and allows AAA1mediated, ATP-induced MT release ${ }^{42}$.
The stalk mediates this communication between the MD and the MTBD through shifts in the positions of the stalk helices relative to one another: coiled-coil 1 (CC1) and coiled-coil 2 (CC2) (Fig. 1b) slide against each other (up to $4.9 \AA$ Å), assuming different stalk registries, which correspond to different MTbinding strengths $s^{24,37,38,44}$. The stalk-helix registries have been shown to influence both dynein-MT-binding affinity and MD ATPase activities ${ }^{24,37,38}$. In solution, the stalk helices predominantly assume a low-affinity registration called the $\beta$ registration ${ }^{38,45}$. However, after MT binding, the stalk helices slide into the high-affinity a registration ${ }^{38,46}$. In the presence of applied mechanical tension, dynein assumes the strong MTbinding a registry under backward load, while forward load induces the recently discovered $\gamma$ registry with intermediate MT-binding strength ${ }^{24}$. The stalk assumes the $\beta$ registry upon AAA1-ATP binding 24 , promoting dissociation of the MTBD from the MT.

Adding complexity to the interactions among AAA1, AAA3, dynein MTBDs, and external and intramolecular tension is evidence that AAA4 may have a regulatory role. When ATP hydrolysis by AAA4 is prevented, dynein processivity increases two-fold and MT-binding affinity increases five-fold ${ }^{47}$. However, blockage of AAA4-ATP hydrolysis reduces dynein velocity only slightly ${ }^{47}$, in contrast to prevention of AAA1-ATP or AAA3-ATP hydrolysis, which dramatically effect velocity (prevention of AAA1 hydrolysis makes the motor immobile $)^{47,48}$. In addition, work on Dictyostelium dynein has shown that blockage of AAA4-ATP binding reduces MT-gliding velocity by $\sim 60 \%$ while blockage of AAA3-ATP binding reduces velocity by $\sim 95 \%{ }^{48}$. It was therefore commonly assumed that AAA4 played a relatively minor role in regulating dynein's mechanochemical stepping cycle, while AAA1 and AAA3 were the key regulators.

Here, we combine mutagenesis with single-molecule fluorescence and optical tweezers-based force measurements ${ }^{24,49-52}$ to demonstrate that AAA4 is a major regulator of dynein motility. We show that while blockage of AAA4-ATP hydrolysis reduces the speed of S. cerevisiae dynein motion only a minimal degree, preventing AAA4-ATP binding abolishes dynein motility completely. We demonstrate that this effect is due to AAA4 regulation of the linker, showing that nucleotide-binding to AAA4 is required for the linker to undock from the MD and perform its priming stroke. Through this mechanism, AAA4 also governs the buttress-mediated sliding of the stalk helices, enabling the stalk to slide from the $\gamma$ to the $\beta$ registration. We further elucidate the interplay between the three active AAA + ATPase domains. We find that blocking AAA4-ATP binding induces the $\gamma$ registry independent of the direction of applied tension if AAA1 is bound to ATP but irrespective of the nucleotide state of AAA3. However, when AAA4 is bound to ATP, the gating of AAA1 by AAA3 prevails, revealing that AAA4 gates the activities of AAA3 and explaining why the effect of preventing ATP hydrolysis by AAA4 on dynein velocity is so mild. Thus, AAA1, 3 , and 4 , the three active ATPases of the MD, work together to regulate dynein's cyclic MT-interactions.

\section{Results}

AAA4-ATP binding is required for dynein motion and force generation. To determine the importance of AAA4 in regulating dynein motion, we used a single-molecule fluorescence motility assay ${ }^{51-53}$ to define the effects of ATP-binding mutations (K/A mutation in the Walker A motif ${ }^{22,54}$ ) and ATPhydrolysis mutations (E/Q mutation in the Walker B motif ${ }^{54,55}$ ) in AAA3 and AAA4 on dynein motility. We introduced E/Q and K/A mutations in Dyn $1_{331 \mathrm{kDa}}$, a minimal $S$. cerevisiae MD containing the linker, AAA + ring, stalk, buttress and 
a Tail Motor domain

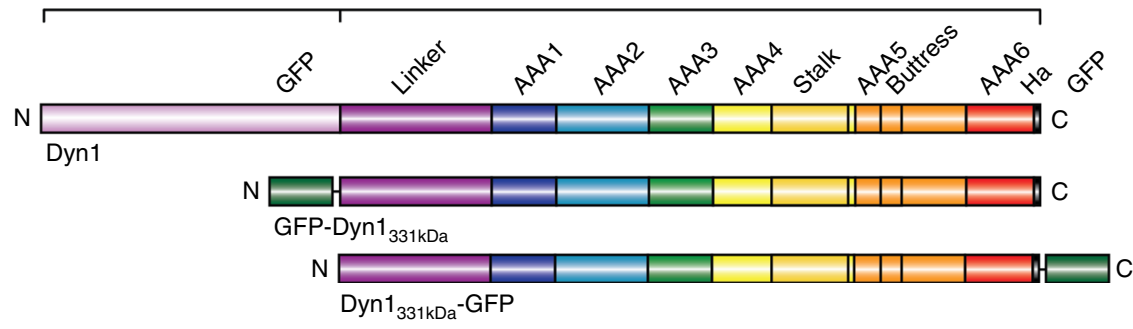

b

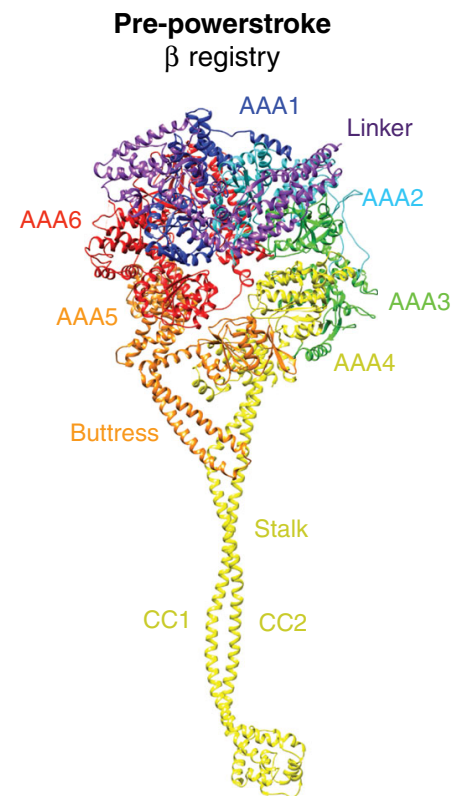

Post-powerstroke $\alpha$ and $\gamma$ registries

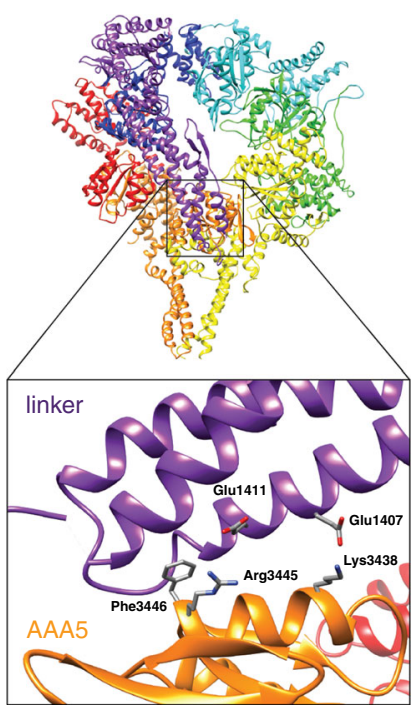

Fig. 1 Cytoplasmic dynein domain organization and pre-powerstroke and post-powerstroke states in relation to the stalk-helix registrations of the dynein motor domain (MD). a Organization of the full-length cytoplasmic dynein heavy chain (HC) (a.a. 1-4092) and the tail-truncated monomeric constructs, GFP-Dyn1 $1_{331 \mathrm{kDa}}$ and Dyn1 $1_{331 \mathrm{kDa}}$-GFP (a.a. 1219-4092). b Dynein MD structure in the pre-powerstroke state (ADP.Vi, Homo sapiens cytoplasmic dynein-2; PDB entry $4 \mathrm{RH} 7^{60}$ ). The linker is bent and close to AAA2 (left), and the stalk helices assume the weak microtubule (MT)-binding $\beta$ registry as a result of the undocked linker ${ }^{24}$. c Post-powerstroke state (Apo, S. cerevisiae dynein; PDB entry 4W8F62). The linker is straight and docked on AAA5, and the stalk helices assume the strong MT-binding $\alpha$ registry or the $\gamma$ registry with intermediate MT-binding strength. Interactions between hydrophobic linker residues (E1407 and E1411) and highly conserved AAA5 residues (F3446, R3445, and K3438) facilitate docking of the linker N-terminus on AAA5 ${ }^{62}$ (PDB entry $4 \mathrm{~W} 8 \mathrm{~F}^{63}$ ) (inset).

MTBD (this construct retains its motor activities $42,52,56$ and is equivalent to the Dictyostelium discoideum MD used in key biochemical studies ${ }^{19,22,37,39}$ ). We then dimerized the singleheaded GFP-Dyn $1_{331 \mathrm{kDa}}$ mutants using an antibody against the $\mathrm{N}$-terminal $\mathrm{GFP}^{24}$ and studied the motors using total internal reflection fluorescence (TIRF) microscopy ${ }^{51,52}$.

Using this approach, we first confirmed that blocking AAA3ATP binding or hydrolysis significantly reduced velocities (11.6 \pm $0.5 \mathrm{~nm} / \mathrm{s}$ and $4.8 \pm 0.3 \mathrm{~nm} / \mathrm{s})$ when compared to WT dynein $(110 \pm$ $2 \mathrm{~nm} / \mathrm{s}$ ) (Fig. 2a, b) $43,47,48,57$, while preventing ATP hydrolysis by AAA4 had only minor effects on dynein velocity ${ }^{47}$ (velocity reduced to $89 \pm 3 \mathrm{~nm} / \mathrm{s}$ ), consistent with previous reports (Fig. 2a, b). In contrast, blocking ATP binding to AAA4 in the AAA4 K/A single mutant completely abolished dynein motion (as did blocking ATP hydrolysis by both AAA3 and AAA4 in the AAA3 E/Q + AAA4 E/Q double mutant) (Fig. 2a). Thus, ATP binding to AAA4 is required for dynein motility.

To further assess the effects of ATP-binding and/or ATPhydrolysis mutations on dynein motion under load, we created homodimeric full-length dynein mutants carrying either the AAA3 E/Q, AAA3 K/A, or AAA4 E/Q mutations and used an optical tweezers setup ${ }^{49,50,58}$ to determine their force-generation capabilities (AAA4 K/A cannot generate motion and therefore cannot be tested in an optical trap). While WT yeast dynein generates $4.5 \mathrm{pN}^{59}$ (Fig. 2e), the AAA3 E/Q and AAA3 K/A mutants exhibit $\sim 60 \%$ and $\sim 50 \%$ reduced stall forces (1.8 and $2.2 \mathrm{pN}$, respectively). Consistent with the minor effects on zeroload motility (Fig. 2a, b), the AAA4 E/Q mutant shows only a $\sim 15 \%$ reduced stall force (Fig. 2 e). The relative reductions in force generation of the AAA3 E/Q and AAA4 E/Q mutants ( $~ 60 \%$ and $\sim 15 \%$ ) compared to WT are comparable with the previous measurements on tail-truncated GST-dimerized mutant motors $\left(\sim 40 \%\right.$ and $\sim 20 \%$, respectively) ${ }^{47}$ (note, however, that while the relative changes are comparable, the absolute forces reported for the GST-dimerized mutant motors are overestimated as a result of an unintended electronic low-pass filtering of the trapping data as we recently discussed ${ }^{59}$ ). These results confirm that preventing AAA3-ATP hydrolysis has a more dramatic effect on dynein's ability to generate force than preventing ATP hydrolysis by AAA4. Thus, while our stall-force measurements on the AAA3ATP and AAA4-ATP hydrolysis mutants alone would support a minor regulatory role for AAA4, our single-molecule TIRF studies on the AAA4-ATP binding mutant reveal that AAA4 has a previously unrecognized essential regulatory role. 
a
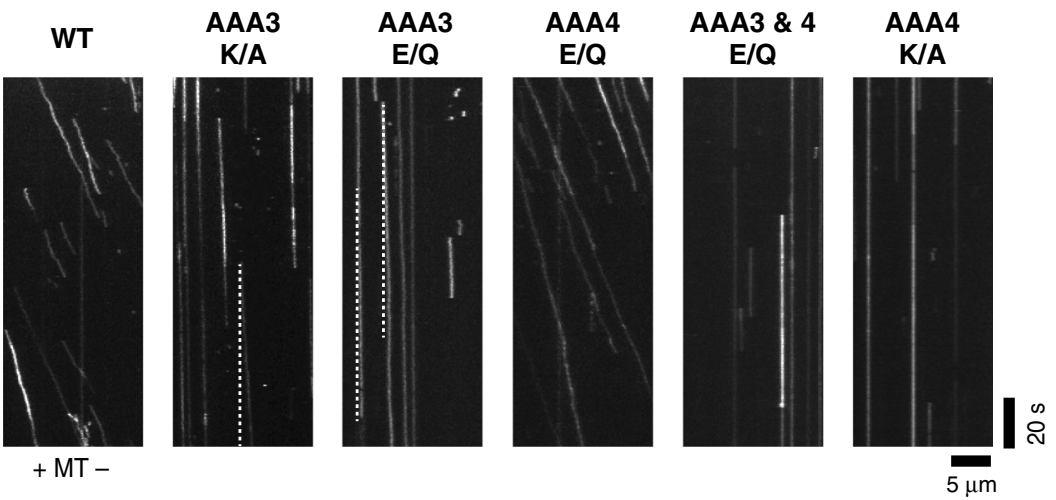

b WT
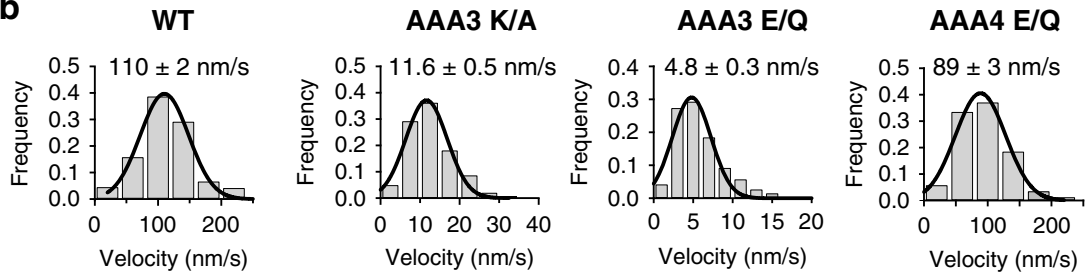

\section{C}

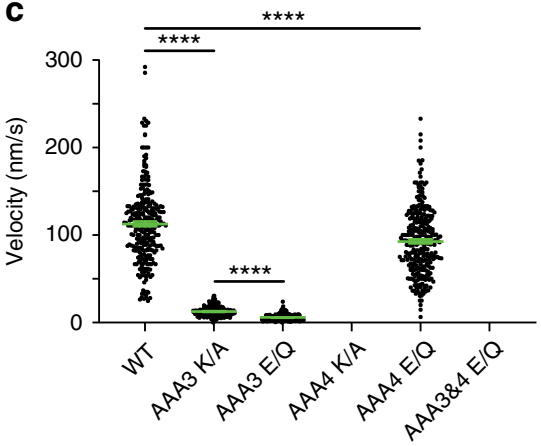

e

WT

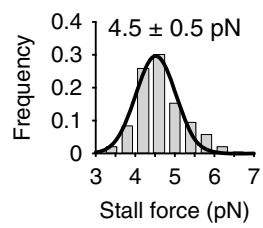

AAA3 K/A

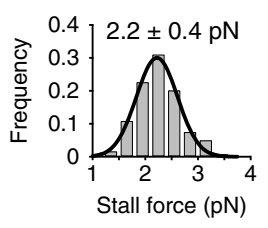

d
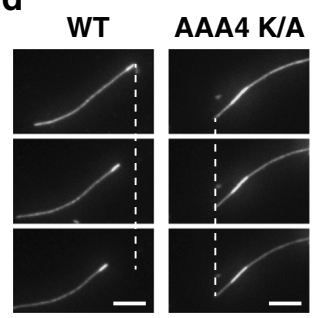

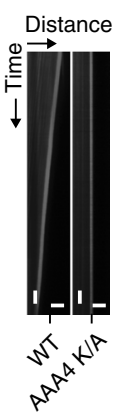

AAA4 E/Q
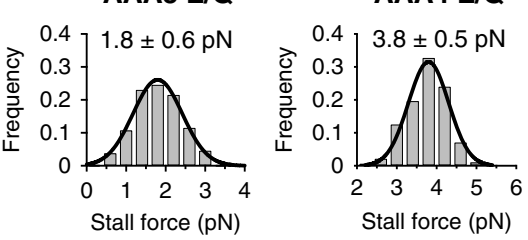

Fig. 2 AAA4-ATP binding is essential for dynein motility. a Antibody-dimerized WT Dyn1 $1_{331 \mathrm{kDa}}$ moves processively in the single-molecule TIRF assay. Diagonal lines in the kymograph represent dimerized molecules that are moving over time. Preventing ATP binding (K/A) or ATP hydrolysis (E/Q) by AAA3 dramatically slows down dynein motion (dashed white lines serve as visual guides to identify slow moving mutants). In contrast, preventing ATP hydrolysis by AAA4 only slightly reduces dynein speed. Strikingly, preventing ATP hydrolysis by both AAA3 and AAA4 or preventing AAA4-ATP binding only, completely abolishes dynein motion (experiments were repeated 3 and 5 times, respectively, with the same results). b Fitting the WT velocity histograms with a Gaussian fit (black line), returns a mean velocity of $110 \pm 2 \mathrm{~nm} / \mathrm{s}$ ( \pm SEM; $N=284$; obtained from $n=6$ independent experiments). Analysis of the AAA3 mutants confirms the low velocities suggested by the kymographs: $11.6 \pm 0.5 \mathrm{~nm} / \mathrm{s}( \pm \mathrm{SEM} ; \mathrm{N}=243 ; n=5)$ for the Dyn1 $_{331 \mathrm{kDa}}$ AAA3 K/A mutant and $4.8 \pm 0.3 \mathrm{~nm} / \mathrm{s}(N=295 ; n=6)$ for the Dyn1 $1_{331 \mathrm{kDa}}$ AAA3 E/Q mutant. The Dyn1 $331 \mathrm{kDa}$ AAA4 E/Q mutant moves with a mean velocity of $89 \pm 3 \mathrm{~nm} / \mathrm{s}(N=307 ; n=5)$. c Comparison of the WT GFP-Dyn1 $331 \mathrm{kDa}$, AAA3 K/A GFP-Dyn1 $331 \mathrm{kDa}$, AAA3 E/Q GFP-Dyn1 $331 \mathrm{kDa}$, and AAA4 E/Q GFP-Dyn1 $331 \mathrm{kDa}$ mean velocities $\left( \pm\right.$ SEM). Statistical significance was determined using an unpaired Welch's $t$ test $\left({ }^{\star \star \star \star} p<0.0001\right)$.

d Kymograph analysis of TMR-labeled and polarity-marked MTs bound to a cover glass via surface-anchored WT Dyn1 $331 \mathrm{kDa}$ and Dyn1 $331 \mathrm{kDa}$ AAA4 K/A in the presence of $1 \mathrm{mM} \mathrm{ATP}$. Left: image sequence showing the gliding of a MT with the bright minus-end lagging, revealing the minus-end-directed activity of WT Dyn1 $1_{331 \mathrm{kDa}}$. Middle: image sequence of an immobile MT bound rigidly to the cover glass via the Dyn1 $331 \mathrm{kDa}$ AAA4 K/A mutant, demonstrating that this mutant is able to bind MTs but incapable of gliding them. Right: kymographs of image sequences of 1,200 images each with representative images shown on the left. The depicted horizontal scale bars correspond to a distance of $5 \mu \mathrm{m}$ and the vertical scale bars to a time period of $10 \mathrm{~s}$. e Histogram of stall forces measured for homodimeric full-length WT GFP-Dyn1 ${ }_{471 \mathrm{kDa}}$ AAA3 K/A GFP-Dyn1 $471 \mathrm{kDa}$, AAA3 E/Q GFP-Dyn1 $471 \mathrm{kDa}$ and AAA4 E/Q GFP-Dyn1 $471 \mathrm{kDa}$ (mean \pm SD). The number of events for each histogram: WT, 189; AAA3 K/A, 119; AAA3 E/Q, $130 ;$ and AAA4 E/Q, 189. Source data are provided as a Source Data file. 

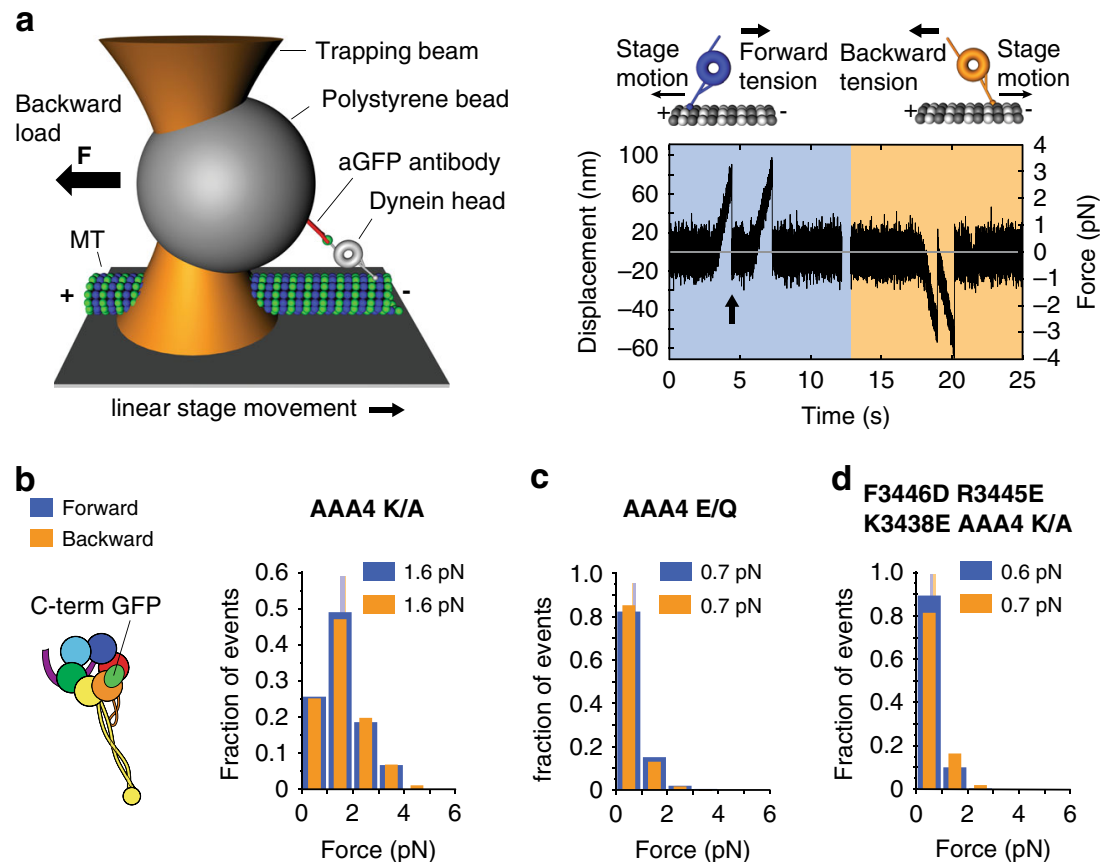

C
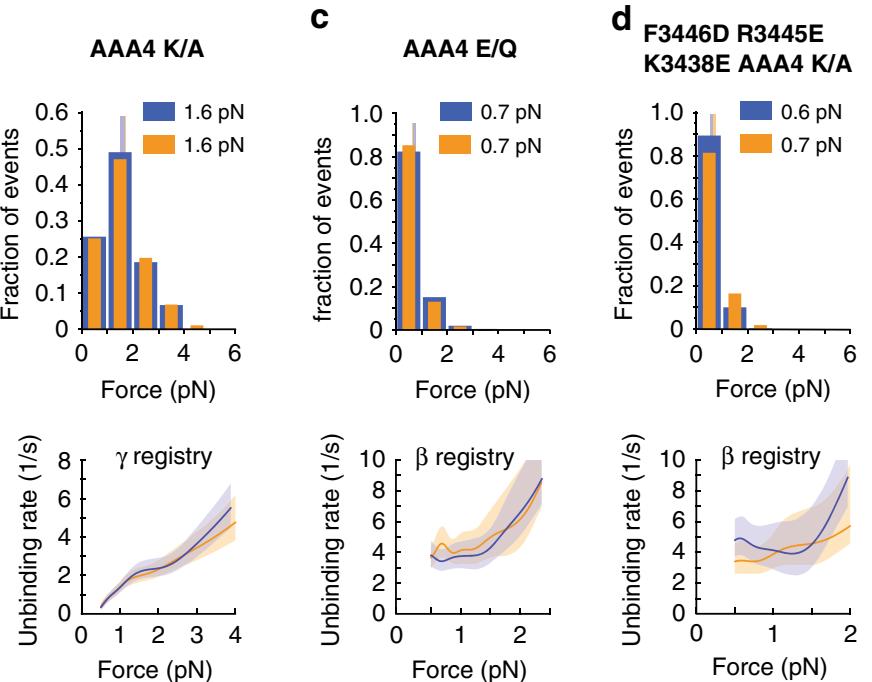

Fig. 3 AAA4-ATP binding controls the linker undocking and the transition from the $\boldsymbol{\gamma}$ registry to the $\boldsymbol{\beta}$ registry. a (Left) A polystyrene bead bearing a dynein motor is held in an optical trap as the microscope stage sweeps back and forth parallel to a MT (not to scale). (Right) Position (force) vs. time for the AAA4 K/A Dyn1 $331 \mathrm{kDa}$-GFP mutant in the presence of $1 \mathrm{mM}$ ATP. Orange and blue shaded areas show periods of applied backward and forward tension, respectively (loading rate: $5.6 \mathrm{pN} / \mathrm{s} ; k=0.036 \mathrm{pN} / \mathrm{nm}, v_{\mathrm{stage}}=156 \mathrm{~nm} / \mathrm{s}$ ). After the motor binds the MT, it pulls the bead out of the trap. Force on the motor increases until the dynein-MT bond ruptures at the "unbinding force" (arrow), here $\sim 3$ pN. b (Left) Schematic of dynein with GFP fused to the C-terminus. (Right) Normalized histograms of primary forward and backward unbinding forces of the AAA4 K/A Dyn1 $331 \mathrm{kDa}$-GFP mutant in the presence of $1 \mathrm{mM}$ ATP. The mean values are noted. Tall vertical bands represent $95 \% \mathrm{Cls}$ of the means (forward: [1.5, 1.7] pN, backward: [1.5, 1.7] pN), which were estimated by bootstrapping 4000 samples. (Bottom) Unbinding rate vs. force derived from the data above. The shaded areas are $95 \% \mathrm{Cls}$ for the mean rates, which were also estimated by bootstrapping. c Same as b, but for the AAA4 E/Q mutant (95\% Cls [0.64, 0.70] and [0.64, 0.71] pN). d Same as in b, but for the F3446D R3445E K3438E AAA4 K/A-Dyn1 $331 \mathrm{kDa}$-GFP mutant (95\% Cls $[0.63,0.7]$ and $[0.65,0.72] \mathrm{pN})$. The number of events in the forward and backward directions: (b) $(269,278),(\mathbf{c})(409,429)$, and (d) $(236,151)$. Source data are provided as a Source Data file.

ATP binding to AAA4 is required for dynein's priming stroke. The finding that blocking AAA4-ATP binding completely prevents dynein motion (Fig. 2a) surprised us, given the previous work on Dictyostelium dynein that showed that the single-headed AAA4 K/A mutant was able to power MT-gliding along coverslip surfaces at $\sim 40 \%$ of the velocity of WT dynein ${ }^{48}$. In contrast, we did not observe MT-gliding by our single-headed S. cerevisiae AAA4 K/A mutant, while single-headed WT dynein moved MTs smoothly (Fig. 2d). We next sought to understand how the AAA4 mutation prevents dynein motion. For dynein to take a forward step, ATP must bind to AAA1, which causes dissociation of the linker from the rear head AAA5 domain ${ }^{17,60}$ (Fig. 1b, c), transition of the stalk helices from the $\gamma$ registry into the weak MT-binding $\beta$ registry ${ }^{24}$, followed by MT release. Linker undocking from AAA5 and subsequent rear head dissociation from the MT are required for the linker to complete its priming stroke $^{24}$. We hypothesized that AAA4 might regulate linker undocking from AAA5, either directly, due to its proximity to the linker docking site, or indirectly, through allosterically regulating AAA1 and/or AAA3 activity.
Our previous work demonstrated that linker undocking from AAA5 is required for the transition from the $\gamma$ registry into the $\beta$ registry $^{24}$ (Fig. 1c). We therefore tested how the AAA4 K/A and AAA4 E/Q mutations affected stalk registry under load by determining dynein-MT binding strengths as a function of the direction of applied tension using optical tweezers, as we have done before ${ }^{24,42}$. In this assay, a polystyrene bead bearing a dynein MD is held in an optical trap as the microscope stage sweeps back and forth parallel to a MT (Fig. 3a, left). The motor binds the MT, pulling the bead out of the trap center. The force on the motor increases until the dynein-MT bond ruptures at the "unbinding force" (Fig. 3a, right). Converting the measured unbinding forces into force-dependent unbinding rates (using a theoretical framework introduced by Olga Dudko and colleagues $^{61}$ and further adapted by us to take into account the compliance of the motor ${ }^{24}$ ) then allows the assignment of stalk registrations. For example, in the absence of nucleotides (apo state), a WT motor domain assumes the a registration (strong MT binding state) under backward load, indicated by an average unbinding force of $\sim 3 \mathrm{pN}$, and assumes the $\gamma$ 
Table 1 Relation of nucleotide condition, pulling position, AAA + ATPase mutations and stalk-helix registrations.

\begin{tabular}{|c|c|c|c|}
\hline $\begin{array}{l}\text { Nucleotide } \\
\text { condition }^{\star}\end{array}$ & Dyn1 $_{331 \mathrm{kDa}}$ construct & $\begin{array}{l}\text { Stalk registry } \\
\text { backward load/ } \\
\text { forward load }\end{array}$ & Summary \\
\hline & Pulling position: C-terminus & & \\
\hline ATP & $\mathrm{WT}^{42}$ & $\beta / \beta$ & registration under $\mathrm{C}$-terminal tension. \\
\hline ATP & AAA4 E/Q & $\beta / \beta$ & Gating of AAA1 by AAA3 prevails if AAA4 is bound to ATP. \\
\hline ATP & $\mathrm{AAA3} E / \mathrm{Q}^{42}$ & $\alpha / \gamma$ & \\
\hline \multirow[t]{2}{*}{ ATP } & $\begin{array}{l}\text { F3446D R3445E K3438E } \\
\text { AAA4 K/A }\end{array}$ & $\beta / \beta$ & require functional interactions between the linker and AAA5 \\
\hline & Pulling position: $\mathbf{N}$-terminus & & \\
\hline Apo & $W T^{24,42}$ & $\alpha / \gamma$ & In the presence of ATP and N-terminal tension, dynein samples \\
\hline ATP & $\mathrm{WT}^{42}$ & $\beta, \alpha / \beta, \gamma$ & $\begin{array}{l}\text { through strong, intermediate and weak MT-binding states as a } \\
\text { result of tension-induced effects on the AAA1 nucleotide- } \\
\text { binding site }{ }^{42} \text {. }\end{array}$ \\
\hline ATP & $\begin{array}{l}\mathrm{AAA} 1 \mathrm{E} / \mathrm{Q}+\mathrm{AAA3} \mathrm{E} / \mathrm{Q}+ \\
\mathrm{AAA} 4 \mathrm{E} / \mathrm{Q}\end{array}$ & $\beta / \beta$ & \\
\hline ATP & $\begin{array}{l}\mathrm{AAA} 1 \mathrm{E} / \mathrm{Q}+\mathrm{AAA} 3 \mathrm{E} / \mathrm{Q}+ \\
\mathrm{AAA} 4 \mathrm{~K} / \mathrm{A}\end{array}$ & $\gamma / \gamma$ & \\
\hline ATP & $\mathrm{AAA} 1 \mathrm{E} / \mathrm{Q}+\mathrm{AAA} 3 \mathrm{~K} / \mathrm{A}^{42}$ & $\alpha / \gamma$ & Even when the AAA3-based gate on AAA1 is closed, the $\alpha$ \\
\hline ATP & $\begin{array}{l}\mathrm{AAA} 1 \mathrm{E} / \mathrm{Q}+\mathrm{AAA} 3 \mathrm{~K} / \mathrm{A}+ \\
\mathrm{AAA} 4 \mathrm{E} / \mathrm{Q}\end{array}$ & $\alpha / \gamma$ & registration can only be induced if AAA4 is bound to nucleotide. \\
\hline ATP & $\begin{array}{l}\text { AAA1 } E / Q+A A A 3 K / A+ \\
\text { AAA4 K/A }\end{array}$ & $\gamma / \gamma$ & \\
\hline ATP & $\mathrm{AAA} 1 \mathrm{E} / \mathrm{Q}+\mathrm{AAA} 4 \mathrm{~K} / \mathrm{A}$ & $\gamma / \gamma$ & \\
\hline ATP & AAA1 $K / A^{42}$ & $\alpha / \gamma$ & Blocking AAA1-ATP binding has a dominant effect on stalk \\
\hline ATP & $\mathrm{AAA} 1 \mathrm{~K} / \mathrm{A}+\mathrm{AAA} 4 \mathrm{E} / \mathrm{Q}$ & $\alpha / \gamma$ & registries and induces the $\alpha$ registry under backward load and \\
\hline ATP & $\mathrm{AAA} 1 \mathrm{~K} / \mathrm{A}+\mathrm{AAA} 4 \mathrm{~K} / \mathrm{A}$ & $\alpha / \gamma$ & $\begin{array}{l}\text { the } \gamma \text { registry under forward load irrespective of the nucleotide } \\
\text { state of AAA4. }\end{array}$ \\
\hline
\end{tabular}

registration under forward load with an average unbinding force of $\sim 1.6 \mathrm{pN}^{24,42}$. In contrast, if the motor is locked in the AAA1 ATP-bound state (AAA1 E/Q mutation), the motor assumes the $\beta$ registration in both directions, exhibiting an average unbinding force of $\sim 0.7 \mathrm{pN}$ in both directions ${ }^{24,42}$ (as previously discussed ${ }^{24}$, the measured forces dependent on the chosen experimental conditions including the loading rate that the dynein-MT bound experiences). We therefore performed unbinding force measurements in the presence of ATP using dynein constructs bearing the AAA4-ATP binding or hydrolysis mutation, and hypothesized that if ATP binding to AAA4 is required for linker undocking from AAA5, the stalk would also be unable to transition from the $\gamma$ to the $\beta$ registration.

We used Dyn $1_{331 \mathrm{kDa}}$ constructs bearing a C-terminal GFP (Fig. 1a) for the attachment to anti-GFP antibody coated optical trapping beads (Fig. 3a). Dyn $1_{331 \mathrm{kDa}-\mathrm{GFP}}$ contains the linker, $\mathrm{AAA}+$ ring, stalk, buttress and MTBD (Fig. 1a), and the linker of this construct can freely assume the pre-powerstroke and postpowerstroke states in the absence of applied tension (Fig. 1b, c). Under C-terminus-applied tension, ATP binding to AAA1 of a WT construct causes dissociation of the linker and the expected transition of the stalk helices from the $\gamma$ to the $\beta$ registry ${ }^{24}$ (Table 1).

We determined the MT-binding strengths of both AAA4 mutants under C-terminus applied tension and compared their behavior with that of WT Dyn $1_{331 \mathrm{kDa}}$ with the stalk helices cross-linked (CL) into different registries (cross-linked constructs were generated previously by introducing cysteines in the outgoing (CC1) and return (CC2) a-helices of the dynein stalk for reversible disulfide cross-linking, in order to lock Dyn $1_{331 \mathrm{kDa}}$ into fixed stalk registries ${ }^{24}$ ). In the presence of $1 \mathrm{mM}$ ATP, the AAA4 K/A mutant with the C-terminal GFP exhibited indistinguishable behavior from the Dyn $1_{331 \mathrm{kDa}}$ mutant with the cross-linked $\gamma$ registry, Dyn $1_{331 \mathrm{kDa}}-\gamma$ CL (Fig. $3 \mathrm{~b}$ and Tables 1,2 ). In contrast, the AAA4 E/Q mutant in the presence of $1 \mathrm{mM}$ ATP behaved similarly to $D y n 1_{331 \mathrm{kDa}}-\beta \mathrm{CL}$, the $\mathrm{Dyn} 1_{331 \mathrm{kDa}}$ mutant with the cross-linked $\beta$ registry (Fig. $3 c$ and Tables 1,2 ). These results demonstrate that ATP binding to AAA4 is required for transition of the stalk helices into the $\beta$-registry, supporting the hypothesis that ATP binding to AAA4 is required for linker dissociation from AAA5 and subsequent transition of the stalk helices into the $\beta$-registry.

To directly test whether the inability of the AAA4 K/A mutant stalk helices to slide was indeed due to prevention of linker undocking from AAA5, we mutated three conserved residues in the AAA5 linker-docking site that have been previously shown to be critical for the docking of the linker to AAA5 ${ }^{62}$ (F3446D, R3445E, and K3438E) (Fig. 1c). We anticipated that this mutant would assume the $\beta$ registry despite having AAA4-ATP binding blocked. Indeed, the F3446D R3445E K3438E AAA4 K/A Dyn $1_{331 \mathrm{kDa}}$ quadruple mutant exhibited statistically indistinguishable behavior from the Dyn $1_{331 \mathrm{kDa}}-\beta$ CL mutant (Fig. $3 \mathrm{~d}$ and Tables 1,2 ), confirming our hypothesis that blocking AAA4ATP binding prevents transition into the $\beta$-registry by preventing linker undocking. 


\section{Table 2 Results of statistical comparisons for various measured unbinding force histograms.}

\section{Experiment $1(\mathrm{pN})$, mean $[\mathrm{Cl}]$}

AAA4 K/A Dyn1 $331 \mathrm{kDa}$-GFP $1 \mathrm{mM}$ ATP forward

$1.6[1.5,1.7]$

AAA4 K/A Dyn1 $331 \mathrm{kDa}$-GFP $1 \mathrm{mM}$ ATP backward

$1.6[1.5,1.7]$

AAA4 E/Q Dyn1 $331 \mathrm{kDa}-$ GFP $1 \mathrm{mM}$ ATP forward

$0.7[0.6,0.7]$

AAA4 E/Q Dyn1 $331 \mathrm{kDa}-$ GFP $1 \mathrm{mM}$ ATP backward

$0.7[0.6,0.7]$

F3446D R3445E K3438E AAA4 K/A-Dyn1331kDa-GFP 1 mM ATP forward

$0.6[0.6,0.7]$

F3446D R3445E K3438E AAA4 K/A-Dyn1 $331 \mathrm{kDa}$-GFP 1 mM ATP backward

$0.7[0.7,0.7]$

AAA1 E/Q + AAA4 E/Q-GFP-Dyn1331kDa $1 \mathrm{mM}$ ATP forward

$0.7[0.6,0.7]$

AAA1 E/Q + AAA4 E/Q-GFP-Dyn1331kDa $1 \mathrm{mM}$ ATP backward

$0.7[0.6,0.7]$

AAA1 E/Q + AAA3 E/Q + AAA4 E/Q-GFP-Dyn1331kDa 1 mM ATP forward

$0.7[0.6,0.7]$

AAA1 E/Q + AAA3 E/Q + AAA4 E/Q-GFP-Dyn1 $331 \mathrm{ka} 1 \mathrm{mM}$ ATP backward

$0.7[0.6,0.7]$

AAA1 E/Q + AAA4 K/A-GFP-Dyn1 $331 \mathrm{kDa} 1 \mathrm{mM}$ ATP forward

$1.6[1.4,1.7]$

AAA1 E/Q + AAA4 K/A-GFP-Dyn1 $331 \mathrm{kDa} 1 \mathrm{mM}$ ATP backward

$1.7[1.5,1.9]$

AAA1 E/Q + AAA3 E/Q + AAA4 K/A-GFP-Dyn1 $331 \mathrm{kDa} 1 \mathrm{mM}$ ATP forward

$1.5[1.4,1.7]$

AAA1 E/Q + AAA3 E/Q + AAA4 K/A-GFP-Dyn1 $331 \mathrm{kDa} 1 \mathrm{mM}$ ATP backward

$1.6[1.5,1.7]$

AAA1 E/Q + AAA3 K/A + AAA4 K/A-GFP-Dyn1 $1_{331 \mathrm{kDa}} 1 \mathrm{mM}$ ATP forward

$1.5[1.4,1.6]$

AAA1 E/Q + AAA3 K/A + AAA4 K/A-GFP-Dyn1 $1_{331 \mathrm{kDa}} 1 \mathrm{mM}$ ATP backward

$1.5[1.5,1.7]$

AAA1 K/A + AAA4 K/A-Dyn1 $331 \mathrm{kDa}-$ GFP $1 \mathrm{mM}$ ATP forward

$1.6[1.5,1.7]$

AAA1 K/A + AAA4 K/A-Dyn1331kDa-GFP 1 mM ATP backward

$2.7[2.4,3]$

AAA1 K/A + AAA4 E/Q-GFP-Dyn1 $331 \mathrm{kDa} 1 \mathrm{mM}$ ATP forward

$1.6[1.5,1.7]$

AAA1 K/A + AAA4 K/A-GFP-Dyn1 $331 \mathrm{kDa} 1 \mathrm{mM}$ ATP backward

$2.7[2.4,3]$

AAA1 E/Q + AAA3 K/A + AAA4 E/Q-GFP-Dyn1 $1_{331 \mathrm{kDa}} 1 \mathrm{mM}$ ATP forward

$1.6[1.5,1.6]$

AAA1 $\mathrm{E} / \mathrm{Q}+\mathrm{AAA} 3 \mathrm{~K} / \mathrm{A}+\mathrm{AAA} 4 \mathrm{E} / \mathrm{Q}-\mathrm{GFP}-\mathrm{Dyn1}_{331 \mathrm{kDa}} 1 \mathrm{mM}$ ATP backward

$2.6[2.4,2.8]$

AAA3 E/Q + AAA4 E/Q-Dyn1 $331 \mathrm{kDa}$-GFP $1 \mathrm{mM}$ ATP forward

$1.6[1.5,1.7]$

AAA3 E/Q + AAA4 E/Q-Dyn1 331kDa-GFP 1 mM ATP backward

$2.6[2.4,2.9]$
Experiment $2(\mathrm{pN})$, mean $[\mathrm{Cl}]$

Dyn1 $_{331 \mathrm{kDa}-} \gamma \mathrm{CL}$ apo forward ${ }^{24}$

$1.6[1.5,1.7]$

Dyn1 $_{331 \mathrm{kDa}-\gamma} \mathrm{CL}$ apo backward ${ }^{24}$

$1.6[1.6,1.7]$

Dyn1 $_{331 \mathrm{kDa}-}-\beta \mathrm{CL}$ apo forward 24

$0.7[0.6,0.7]$

Dyn1 $_{331 \mathrm{kDa}}-\beta \mathrm{CL}$ apo backward 24

$0.7[0.7,0.8]$

Dyn1 $_{331 \mathrm{kDa}}-\beta \mathrm{CL}$ apo forward 24

$0.7[0.6,0.7]$

Dyn1 $_{331 \mathrm{kDa}}-\beta \mathrm{CL}$ apo backward 24

$0.7[0.7,0.8]$

Dyn1 $_{331 \mathrm{kDa}}-\beta \mathrm{CL}$ apo forward ${ }^{24}$

$0.7[0.6,0.7]$

Dyn1 $_{331 \mathrm{kDa}}-\beta \mathrm{CL}$ apo backward ${ }^{24}$

$0.7[0.7,0.8]$

Dyn1 $_{331 \mathrm{kDa}}-\beta \mathrm{CL}$ apo forward ${ }^{24}$

$0.7[0.6,0.7]$

Dyn1 $_{331 \mathrm{kDa}}-\beta \mathrm{CL}$ apo backward 24

$0.7[0.7,0.8]$

Dyn1 $_{331 \mathrm{kDa}}-\gamma \mathrm{CL}$ apo forward 24

$1.6[1.5,1.7]$

Dyn1 $_{331 \mathrm{kDa}^{-} \gamma} \mathrm{CL}$ apo backward 24

$1.6[1.6,1.7]$

Dyn1 $_{331 \mathrm{kDa}-} \gamma \mathrm{CL}$ apo forward 24

$1.6[1.5,1.7]$

${\text { Dyn } 1_{331 \mathrm{kDa}}-\gamma \mathrm{CL} \text { apo backward }}^{24}$

$1.6[1.6,1.7]$

Dyn1 $_{331 \mathrm{kDa}}-\gamma \mathrm{CL}$ apo forward ${ }^{24}$

$1.6[1.5,1.7]$

Dyn1 $_{331 \mathrm{kDa}-} \gamma \mathrm{CL}$ apo backward 24

$1.6[1.6,1.7]$

Dyn1 $_{331 \mathrm{kDa}-} \gamma \mathrm{CL}$ apo forward ${ }^{24}$

$1.6[1.5,1.7]$

Dyn1 $_{331 \mathrm{kDa}^{-} \alpha \mathrm{CL} \text { apo backward }} 24$

$2.7[2.5,3]$

Dyn1 $_{331 \mathrm{kDa}-} \gamma \mathrm{CL}$ apo forward 24

$1.6[1.5,1.7]$

Dyn $_{331 \mathrm{kDa}}-\alpha \mathrm{CL}$ apo backward 24

$2.7[2.5,3]$

Dyn1 $_{331 \mathrm{kDa}}-\gamma \mathrm{CL}$ apo forward 24

$1.6[1.5,1.7]$

Dyn1 $_{331 \mathrm{kDa}}-\alpha \mathrm{CL}$ apo backward 24

$2.7[2.5,3]$

Dyn1 $_{331 \mathrm{kDa}}-\gamma \mathrm{CL}$ apo forward 24

$1.6[1.5,1.7]$

Dyn1 $_{331 \mathrm{kDa}}-\alpha \mathrm{CL}$ apo backward 24

$2.7[2.5,3]$
$\boldsymbol{P}_{\mathbf{m}}$

$<0.75$

$<0.65$

$<0.2$

$<0.4$

$<0.22$

$<0.45$

$<0.83$

$<0.38$

$<0.83$

$<0.3$

$<0.96$

$<0.66$

$<0.62$

$<0.33$

$<0.32$

$<0.53$

$<0.63$

$<0.8$

$<0.63$

$<0.79$

$<0.84$

$<0.53$

$<0.8$

$<0.7$
AAA4-based block on linker undocking is independent of AAA3. Previous work demonstrates that ATP binding to AAA1 is required for linker undocking ${ }^{23}$ and that AAA3 gates AAA1 activity, so that AAA1-ATP binding induces undocking of the linker and the weak MT-binding $\beta$ registration only when AAA3 is in the post-hydrolysis state ${ }^{42,43}$. However, when tension is applied to the linker, ATP binding to AAA3 is sufficient to "open" the gate ${ }^{42}$. The conclusion that AAA3 affects the linker indirectly via AAA1 is supported by the MT-binding behavior of the AAA3 E/Q mutant: blocking AAA3-ATP hydrolysis yields the $\gamma$ registry under forward load and the a registry under backward load 24,42 , a behavior identical to that of the AAA1-ATP binding (AAA1 K/A) mutant and the WT motor in the apo state 24,42 (Table 1). In contrast, blocking AAA4-ATP binding yields the $\gamma$ registry in both directions (Fig. 3a, b). This indicated that unlike AAA3, which exerts regulatory control of linker undocking indirectly through AAA1, ATP binding to AAA4 regulates linker undocking and stalk registry changes through a different pathway. We hypothesized, given AAA4's proximity to the linker docking site at AAA5, that AAA4 may regulate linker undocking and stalk registry changes directly.

To provide further evidence that the block imposed on linker undocking by AAA4 is direct and to elucidate the hierarchy between the AAA4 K/A-induced block on the linker transition from a docked to an undocked state and AAA1 and AAA3 function, we employed novel double and triple AAA + ATPase mutants.

We first confirmed that the AAA4 K/A-induced block on linker undocking was independent of AAA3. As mentioned, under linker-applied tension, the binding of ATP to AAA3 opens the gate and AAA1-ATP binding induces the $\beta$ registry ${ }^{24,42}$. Thus, in the presence of $1 \mathrm{mM}$ ATP, the AAA1 E/Q single mutant 
and the AAA1 E/Q + AAA3 E/Q double mutant assume the $\beta$ registry in both directions, while the AAA1 K/A single mutant and the AAA1 E/Q + AAA3 K/A double mutant assume the $\gamma$ registry under forward load and the a registry under backward load $^{24,42}$ (Table 1). As ATP binding to AAA4 is required for the transition into the $\beta$ registry (Fig. $3 \mathrm{~b}, \mathrm{c}$ ), we anticipated that the AAA1 E/Q + AAA4 E/Q double mutant and the AAA1 E/Q + AAA3 $\mathrm{E} / \mathrm{Q}+\mathrm{AAA} 4 \mathrm{E} / \mathrm{Q}$ triple mutant would assume the $\beta$ registry in the presence of $1 \mathrm{mM}$ ATP, while blocking AAA4-ATP binding in the double mutant (AAA1 E/Q + AAA4 K/A) and the triple mutant (AAA1 E/Q + AAA3 E/Q + AAA4 K/A) would prevent reduced MT binding and induce the $\gamma$ registry in both directions, in spite of the AAA3 gate being open and AAA1 nucleotide bound. As expected, the AAA1 E/Q + AAA4 E/Q double mutant and the AAA1 E/Q + AAA3 E/Q + AAA4 E/Q triple mutant assumed the $\beta$ registry in the presence of $1 \mathrm{mM}$ ATP (Fig. 4a, b and Tables 1, 2), while the AAA1 E/Q + AAA4 K/ A double mutant and the AAA1 E/Q + AAA3 E/Q + AAA4 K/A triple mutant assumed the $\gamma$ registry in both directions (Fig. $4 \mathrm{c}, \mathrm{d}$ and Tables 1,2).

To test whether blocking ATP binding to AAA4 was also able to induce the $\gamma$ registry when AAA3 was nucleotide-free (when the gate on AAA1 is closed), we explored the MT-binding behavior of the AAA1 E/Q + AAA3 K/A + AAA4 K/A triple mutant. As expected, this triple mutant also assumed the $\gamma$ registry in the presence of $1 \mathrm{mM}$ ATP in both directions (Fig. 4e and Tables 1,2). Thus, the ability of AAA4's nucleotide free state to block changes in MT-binding strength is independent of whether the AAA3 gate is closed or open. In addition, the fact that the AAA1 E/Q + AAA4 K/A double mutant assumed the $\gamma$ registry irrespective of the direction of applied tension (Fig. 4d) demonstrates that the AAA4 K/A mutation also blocked linker undocking even when AAA3 assumed a post-ATP-hydrolysis state (AAA3 of this mutant can bind and hydrolyze ATP and reach the ADP.Pi transition state for some fraction of the dynein cycle $^{43}$, which is sufficient to open the gate on AAA1 so that the AAA1 E/Q single mutant assumes the $\beta$ registry at $1 \mathrm{mM}$ $\mathrm{ATP}^{24,42}$ ). Therefore, AAA4's block on linker undocking is independent of the nucleotide state of AAA3.

AAA4-based regulation of MT-binding strength is dependent on the nucleotide state of AAA1. As discussed, blocking AAA4ATP binding induces the $\gamma$ registry in both MT directions (Fig. 3a, b). In contrast, WT dynein in the absence of nucleotides (apo) and the AAA1 K/A single mutant in the presence of ATP both assume the a registry under backward load ${ }^{24,42}$ (Table 1). Therefore, blocking ATP binding to AAA4 alone has a distinct effect on stalk registry from blocking nucleotide binding to both AAA1 and AAA4, or blocking ATP binding to AAA1 alone. This suggests that while AAA4 regulation of stalk registry is independent of the nucleotide-binding state of AAA3, it is dependent upon the nucleotide-binding state of AAA1.

To confirm this, we generated the AAA1 K/A + AAA4 E/Q and the AAA1 K/A + AAA4 K/A double mutants and performed unbinding-force measurements. As expected, both double mutants assume the $\alpha$ registry under backward load and the $\gamma$ registry under forward load in the presence of ATP (Fig. 5a, b and Tables 1,2). This implies that the effect of ATP binding to AAA4 on linker undocking is secondary to the regulation of linker undocking and stalk sliding by AAA1, since blockade of ATP-binding to AAA4 only impacts stalk registry when AAA1 is nucleotide bound.

AAA3 controls ATP-induced, AAA1-mediated MT release when AAA4 is bound to ATP. Our work demonstrates that AAA4 regulation of linker undocking is independent of AAA3's nucleotide state, but dependent on the nucleotide state of AAA1 (whether the AAA3 gate was opened or closed, the stalk helices assumed the $\gamma$ registry if AAA4 ATP-binding was blocked, but only if AAA1 is ATP bound). The observation that blocking AAA4-ATP hydrolysis has only a minor effect on dynein function (Fig. 2) suggests that AAA4 no longer exerts a dominant role once bound to ATP. Therefore we hypothesized that if AAA4 is nucleotide bound, AAA3 assumes its role in controlling AAA1 function ${ }^{42,43}$

To test this hypothesis, we determined the unbinding behavior of the AAA1 E/Q + AAA3 K/A + AAA4 E/Q triple mutant with the $\mathrm{N}$-terminal GFP. Under N-terminus-applied tension, blocking AAA3-ATP binding closes the gate on AAA1 (AAA1-ATP binding cannot induce MT release) and, as previously stated, results in the $\gamma$ registry under forward load and the a registry under backward load $^{24,42}$ (Table 1). As expected, we found that the AAA1 E/Q + AAA3 K/A + AAA4 E/Q triple mutant in the presence of $1 \mathrm{mM}$ ATP assumed the $\gamma$ registry under forward load and the a registry under backward load (Fig. 6a and Tables 1,2). In addition, the AAA3 E/Q + AAA4 E/Q double mutant with the C-terminal GFP (under C-terminus-applied tension, blocking AAA3-ATP hydrolysis closes the gate on AAA ${ }^{42}$; Table 1 ) also assumed the $\gamma$ registry under forward load and the a registry under backward load in the presence of 1 mM ATP (Fig. $6 \mathrm{~b}$ and Tables 1, 2). Thus, when AAA4 was ATP bound, AAA3 gates the activity of AAA1. Therefore, both AAA4 and AAA3 regulate dynein-MT binding strength, and both their regulatory mechanisms are dependent upon the nucleotide state of AAA1. This dual dominance underscores the intricate complexity and convoluted interactions among dynein's primary and regulatory AAA + domains.

\section{Discussion}

Previous studies suggested that AAA4 has only a minor role in dynein function. Here, we identify AAA4 as a key regulator of dynein motion, demonstrating that ATP binding to AAA4 is necessary for linker undocking and subsequent stalk-helices sliding and MT release. We further elucidate the hierarchy of regulation of MT-binding strength by the active AAA + ATPase domains, demonstrating that nucleotide binding to AAA4 gates the regulatory function of AAA3, which in turn gates AAA1. While AAA3 exerts influence on MT-binding strength through an entirely allosteric mechanism via AAA1, our results suggest that AAA4 regulation of linker undocking and stalk sliding is more complex. The unique stalk-registry profile assumed by the AAA4 K/A mutant (the $\gamma$-registry under forward and backward load) suggests that nucleotide binding to AAA4 may directly influence linker conformation. At the same time, regulation of dynein-MT binding strength by AAA4 is dependent on the nucleotide state of AAA1: ATP binding to AAA4 alone has no effect on stalk registry unless AAA1 is also bound to nucleotide. These findings underscore the intricate complexity and convoluted interactions among dynein's primary regulatory AAA+ domains in dynein's mechanochemical cycle.

We recently reported that the linker controls the buttressmediated sliding of the stalk helices to alter the MT-binding strength of the two MTBDs within dynein ${ }^{24}$. These past results combined with the data presented here suggest that AAA4 has an essential function in controlling dynein's cyclic MT interactions through regulation of linker undocking and subsequent stalkhelices sliding. This conclusion appears to contradict a previous study on Dictyostelium dynein where it was reported that the AAA4 K/A mutation reduced MT-gliding activity by only $60 \%{ }^{48}$. While it is possible that Dictyostelium and S. cerevisiae dynein behave differently in the mutant background, future studies will be required to resolve this discrepancy and to show whether 
a

Forward
Backward

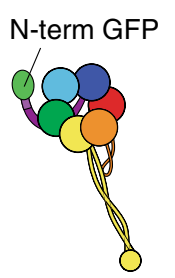

C
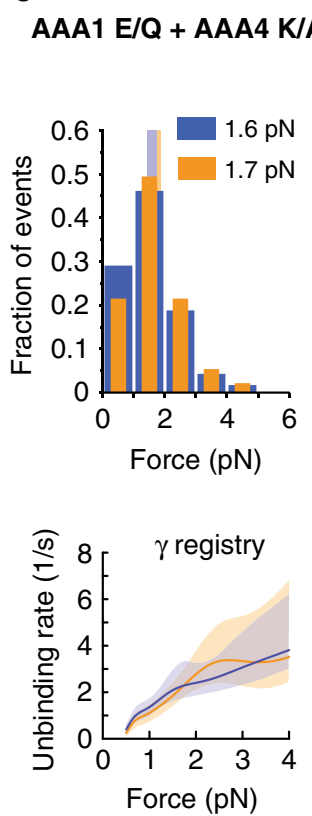

AAA1 E/Q + AAA4 E/Q
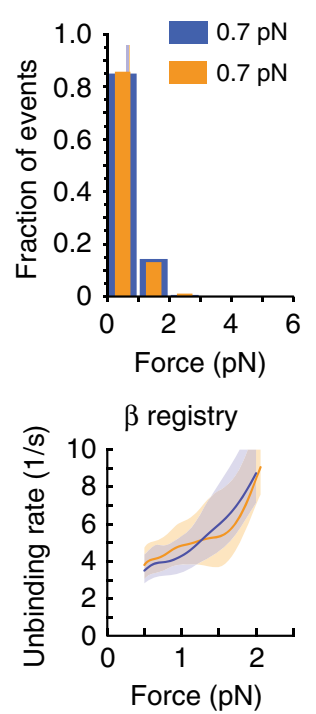

d

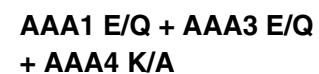
+ AAA4 K/A
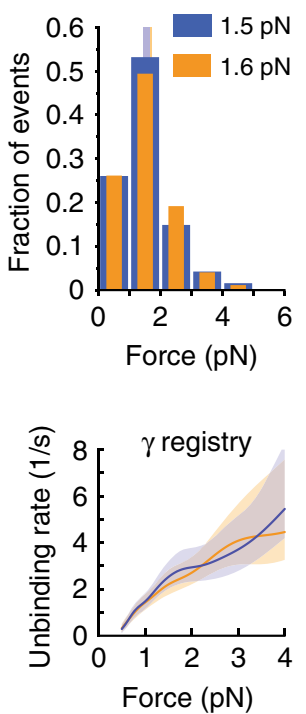

b

\section{AAA1 E/Q + AAA3 E/Q \\ + AAA4 E/Q}
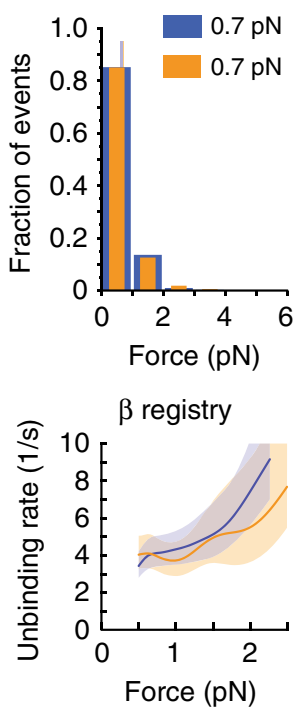

e

AAA1 E/Q + AAA3 K/A

+ AAA4 K/A
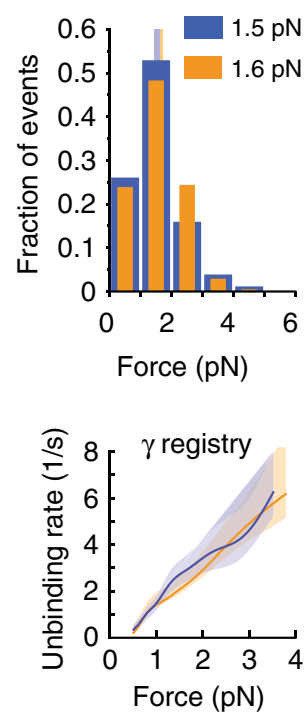

Fig. 4 Blocking AAA4-ATP binding induces the $\boldsymbol{\gamma}$ registry irrespective of the AAA3 nucleotide state. a (Left) Schematic of dynein with GFP fused to the $\mathrm{N}$-terminus. (Right) Histogram of forward (blue) and backward (orange) unbinding forces for the AAA1 E/Q + AAA4 E/Q-GFP-Dyn1 $331 k D a$ double mutant measured in the presence of $1 \mathrm{mM}$ ATP. The respective mean values are noted. The vertical bands represent $95 \% \mathrm{Cls}$ for the means (forward: [0.6, 0.7] $\mathrm{pN}$, backward $[0.6,0.7] \mathrm{pN}$ ). (Bottom) Unbinding rate vs. force derived from the data above. The shaded areas are the $95 \% \mathrm{Cls}$ for the mean rates, which were estimated by bootstrapping. b Same as in $\mathbf{a}$, but for the AAA1 E/Q + AAA3 E/Q + AAA4 E/Q-GFP-Dyn1 $331 \mathrm{kDa}$ triple mutant (95\% Cls [0.6, 0.7] and $[0.6,0.7] \mathrm{pN})$. c Same as in a, but for the AAA1 E/Q + AAA4 K/A-GFP-Dyn1 ${ }_{331 \mathrm{kDa}}$ double mutant $(95 \% \mathrm{Cls}[1.4,1.7]$ and $[1.5,1.9] \mathrm{pN})$. d Same as in a, but for the AAA1 E/Q + AAA3 E/Q + AAA4 K/A-GFP-Dyn1 $331 \mathrm{kDa}$ triple mutant $(95 \%$ Cls $[1.4,1.7]$ and $[1.5,1.7] \mathrm{pN})$. e Same as in a, but for the AAA1 E/Q + AAA3 K/A + AAA4 K/A-GFP-Dyn1 $331 \mathrm{kDa}$ triple mutant (95\% Cls $[1.4,1.6]$ and $[1.5,1.7] \mathrm{pN})$. The number of events in the forward and backward directions: (a) $(321,358),(\mathbf{b})(419,382),(\mathbf{c})(117,93),(\mathbf{d})(188,172)$, and (e) $(257,205)$. Source data are provided as a Source Data file.

AAA4 of mammalian dynein has the same function as AAA4 of $S$. cerevisiae dynein, but mutations in AAA4 that cause malformation of cortical development reinforce the importance of AAA4 in dynein mechanochemistry ${ }^{9}$.

Our single-molecule TIRF studies and the work of others ${ }^{47}$ have shown that the homodimeric AAA4 E/Q mutant moves processively at $\sim 80 \%$ of the velocity of WT dynein (Fig. $2 \mathrm{a}, \mathrm{b}$ ). As the
AAA4 E/Q mutant with the C-terminal GFP assumes the $\beta$ registration in the presence of ATP (Fig. 3c), this result appears to be in conflict with our previous work that demonstrated that the dimerized Dyn $1_{331 \mathrm{kDa}}-\beta$ CL construct with the cross-linked $\beta$ registry cannot move ${ }^{24}$. However, while Dyn $1_{331 \mathrm{kDa}}-\beta \mathrm{CL}$ construct and the AAA4 E/Q mutant with the C-terminal GFP only assume the $\beta$ registry, the AAA4 E/Q mutant with the N-terminal GFP can 

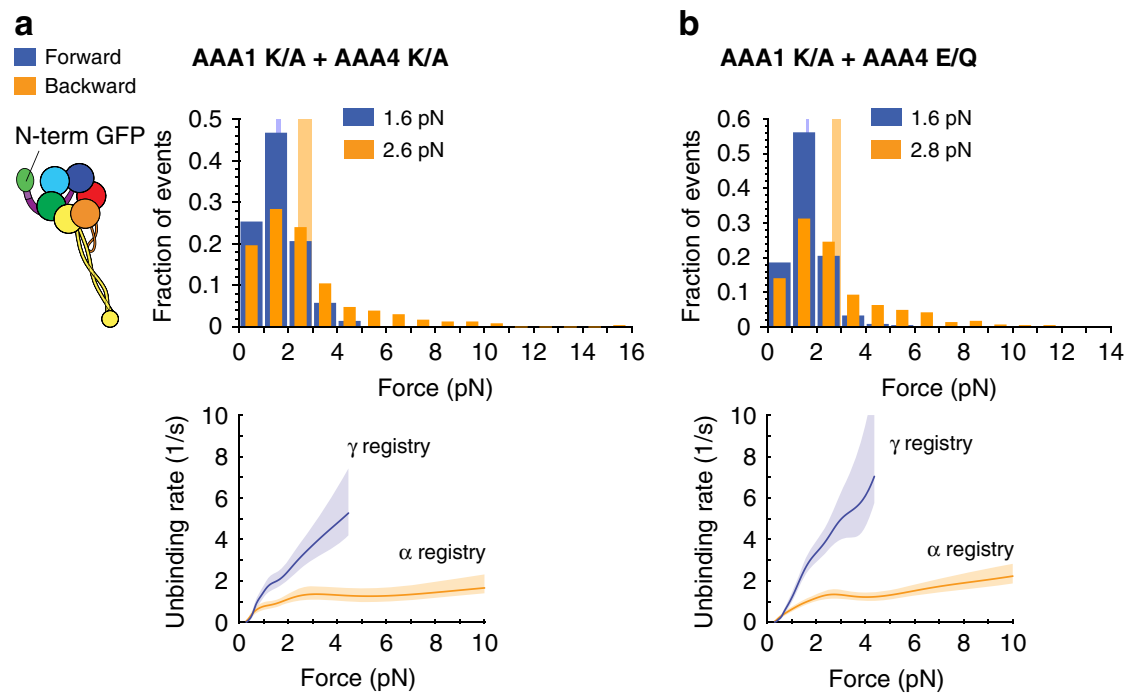

Fig. 5 The effects of AAA4 on dynein-MT binding require AAA1-ATP binding. a (Left) Schematic of dynein with GFP fused to the N-terminus. (Right) Histogram of forward (blue) and backward (orange) unbinding forces for the AAA1 K/A + AAA4 K/A-GFP-Dyn1 $331 \mathrm{kDa}$ double mutant measured in the presence of $1 \mathrm{mM}$ ATP. The respective mean values are noted. The vertical bands represent $95 \% \mathrm{Cls}$ for the means (forward: [1.5, 1.7] pN, backward [2.4, 3] pN). (Bottom) Unbinding rate vs. force derived from the data above. The shaded areas are the $95 \% \mathrm{Cls}$ for the mean rates, which were estimated by bootstrapping. b Same as in $a$, but for the AAA1 K/A + AAA4 E/Q-GFP-Dyn1 ${ }_{331 \mathrm{kDa}}$ double mutant $(95 \% \mathrm{Cls}[1.6,1.7]$ and $[2.6,3] \mathrm{pN})$. The number of events in the forward and backward directions: (a) $(276,229)$ and $(\mathbf{b})(575,569)$. Source data are provided as a Source Data file.

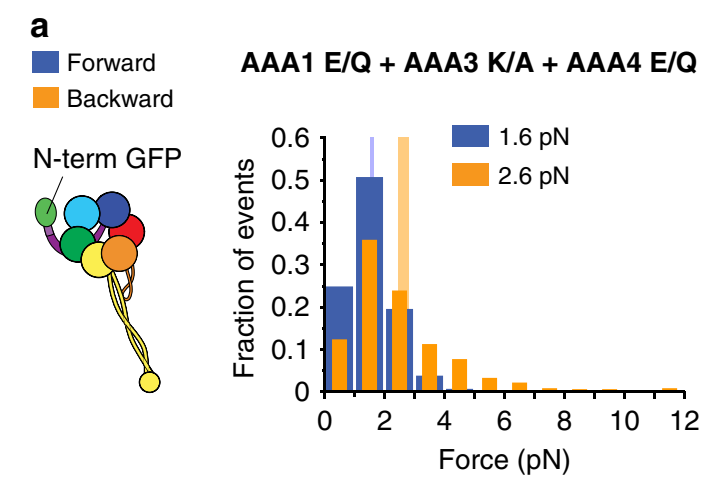

b

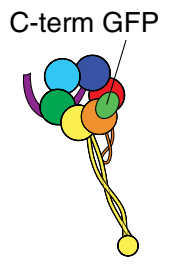

AAA3 E/Q + AAA4 E/Q

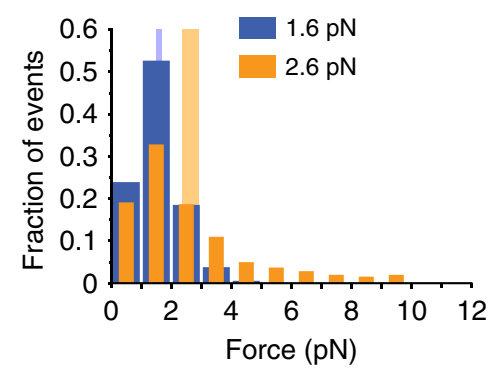

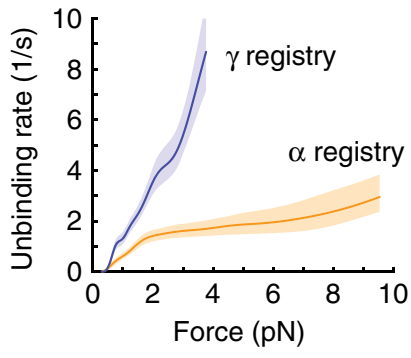

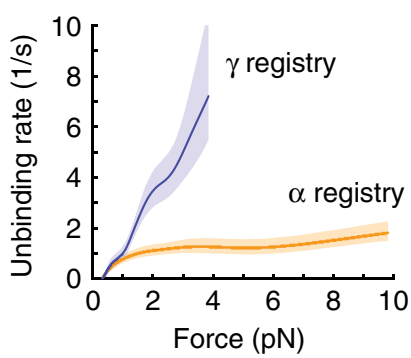

Fig. 6 ATP binding to AAA4 is not sufficient to overcome the AAA3-induced block on AAA1. a Schematic of dynein with GFP fused to the N-terminus (left). Histogram of forward (blue) and backward (orange) unbinding forces for the AAA1 E/Q + AAA3 K/A + AAA4 E/Q-GFP-Dyn1331kDa triple mutant measured under $\mathrm{N}$-terminal tension in the presence of $1 \mathrm{mM}$ ATP (middle). The respective mean values are noted. The vertical bands represent $95 \% \mathrm{Cls}$ for the means (forward: $[1.5,1.6] \mathrm{pN}$, backward $[2.4,2.8] \mathrm{pN}$ ). (Right) Unbinding rate vs. force derived from the data on the left. The shaded areas are $95 \%$ $\mathrm{Cls}$ for the mean rates, which were estimated by bootstrapping. b Same as in $a$, but for the AAA3 E/Q + AAA4 E/Q-Dyn1331kDa-GFP double mutant measured under C-terminal tension (95\% Cls $[1.5,1.7]$ and $[2.4,3] \mathrm{pN})$. The number of events in the forward and backward directions: $(\mathbf{a})(546,451)$ and (b) $(279,234)$. Source data are provided as a Source Data file.

switch between different registrations depending on the nucleotide state of AAA1 and AAA3 (i.e., when tension is applied via the Nterminal linker) (Figs. 4 and 5). Together with our previous work that demonstrated that WT dynein with a C-terminal GFP assumes the $\beta$ registry in the presence of ATP while WT dynein with an $\mathrm{N}$ terminal GFP shows an unbinding behavior that reflects transitions through different stalk registrations ${ }^{42}$ (Table 1), this suggests that tension applied via the linker facilitates the motor's progression 
through its mechanochemical cycle, and explains why the homodimeric AAA4 E/Q mutant would be able to move processively. This conclusion is supported by the work by Clearly and coworkers ${ }^{56}$ and the work of our lab ${ }^{42}$, which has shown that tension applied to the linker gates the ATP-dependent release of dynein from MTs. As the AAA4 E/Q mutant motor domains are linked together via the N-terminal GFPs (using an antibody against GFP, Fig. 2a, b) and as the mutant motor moves processively, this indeed suggests that the AAA4 E/Q mutant can assume different registrations (such as the a registration under backward load when AAA1 assumes the post-hydrolysis state, Fig. 5) if sufficient tension develops during the two-head bound state, while the $\mathrm{Dynl}_{331 \mathrm{kDa}}-\beta$ CL construct cannot.

Our single-molecule TIRF studies further revealed that the AAA3 E/Q + AAA4 E/Q double mutant does not move (Fig. 2), which can be explained by the MT-binding behavior of the mutant. Our unbinding-force studies showed that under Cterminus applied tension, AAA3 E/Q + AAA4 E/Q-Dyn1 ${ }_{331 \mathrm{kDa}^{-}}$ GFP assumes the $\gamma$ registry under forward load and the $\alpha$ registry under backward load in the presence of $1 \mathrm{mM}$ ATP (Fig. 6b). Because both registrations can only be assumed when the linker is docked, the data suggest that the linker is always docked and that the priming stroke never occurs, and thus the motor cannot move. The Dyn1 $1_{331 \mathrm{kDa}^{-}}-\alpha$ CL mutant still moves slowly ${ }^{24}$, which excludes the possibility that the ATP-insensitive MT-binding strength of the AAA3 E/Q + AAA4 E/Q double mutant accounts for its immobility. Intriguingly, the linker of the AAA3 E/Q mutant has also been shown to be locked in the post-powerstroke state in the absence of a load ${ }^{63}$, but the dimerized AAA3 E/Q mutant moves slowly (Fig. 2a, b). This implies that the linker of the AAA3 E/Q mutant can detach under tension and undergo a priming stroke-albeit less efficiently-whereas the AAA3 E/Q + AAA4 E/Q double mutant cannot. Therefore, the interaction strength between the linker and AAA5 could be stronger when ATP hydrolysis is prevented in both regulatory ATPase domains. The observation that the dimerized AAA4 K/A mutant is immotile further supports the premise that blocking AAA4-ATP binding could result in a stabilized, docked linker conformation.

The finding that blocked AAA4-ATP binding induced the $\gamma$ registry independent of both the direction of applied tension and the nucleotide state of AAA3 is intriguing because it suggests that AAA4 can prevent tension-induced and nucleotide-induced changes in the stalk helices. Our data with the F3446D R3445E K3438E AAA4 K/A quadruple mutant showed that the effects of AAA4 on the stalk registry required functional interactions between the linker and AAA5 (Fig. 3d). However, our previous work has also demonstrated that the a registry requires the linker to dock to AAA5 in the apo state and that despite the docked state, directional tension can shift the registries interchangeably between $\alpha$ and $\gamma^{24,42}$. Blocking AAA4-ATP binding locks the $\gamma$ registry in both directions and prevents a change to the a registry under backward load, implying that the linker docks to AAA5 in a way that differs from the docked state required for the a registry. Indeed, the crystal structures in the absence of a nucleotide ${ }^{62}$ (Fig. 1b) and in the presence of $\mathrm{ADP}^{21}$ reveal two distinct post-powerstroke positions. In the apo state, the linker is positioned above the large domain of AAA5 (AAA5L) ${ }^{62}$. In the ADP state, the linker is positioned between AAA5L and the large domain of AAA4 (AAA4L) ${ }^{21}$. Direct linker interaction with AAA4, especially when AAA1 is bound to ATP and AAA4 is nucleotide-free, (Fig. 4d), remains to be shown. Solving the crystal structure of the AAA1 E/Q + AAA4 K/A double mutant (Fig. 4d) or the AAA1 E/Q + AAA3 E/Q + AAA4 K/A triple mutant (Fig. $4 \mathrm{~b}$ ) in the presence of ATP would help to evaluate this possibility.

Our results in Figs. 5 and 6 exemplified again the unique bond that dynein forms with a MT under backward load when the stalk helices assume the a registry. In agreement with our previous work ${ }^{24,42}$, the a registry caused dynein to exhibit "slip-ideal" bonding with the MTs for forces up to $\sim 5-6 \mathrm{pN}$. Faster unbinding was observed for backward forces up to $\sim 2 \mathrm{pN}$ and a constant, force-independent unbinding rate occurred for greater backward forces (Fig. 5). Dynein exhibited slip-bonding up to $4 \mathrm{pN}$ when it assumed the $\gamma$ registry ${ }^{24}$ (Fig. 3, 4). Noting that the movement of full-length $S$. cerevisiae dynein $\left(\mathrm{Dyn}_{471 \mathrm{kDa}}\right)$ ceases at $4.5 \mathrm{pN}^{59}$ ("stall force"), these bond behaviors capture the most relevant force range of dynein when the motor is actively moving forward or stalling (when dynein is forced to move backward, larger forces can occur ${ }^{64}$ ).

While a recent study modeled the apo-state bond behavior of a single $S$. cerevisiae dynein head as an asymmetric slip bond for forces up to $14 \mathrm{pN}^{65}$, a closer look at the measured data shows an excellent agreement with our results for forces up to $\sim 5 \mathrm{pN}$, including the slip bonding up to $\sim 2 \mathrm{pN}$ and an ideal bonding up to $\sim 5 \mathrm{pN}$ under backward load. Our data also suggest an increasing unbinding rate above $\sim 5 \mathrm{pN}$ so that the bond appears best described by an overall slip-ideal-slip behavior for the $0-10$ $\mathrm{pN}$ force range (Figs. 5 and 6), but the rates above $\sim 5 \mathrm{pN}$ that Ezber et al. ${ }^{65}$ reported are somewhat larger than ours. While the differences at larger forces could result from the different unbinding-force assays used ${ }^{24}$, future studies are necessary to accurately determine how the dynein-MT bond behaves under super-stall forces.

In conclusion, our work demonstrates that blocking AAA4ATP binding locks the dynein linker element in the postpowerstroke state and locks the stalk helices in the $\gamma$ registry. Our data further suggests that ATP binding to AAA4 is necessary for the trailing head to undock the linker from AAA4/AAA5, which allows for the transition into the weak MT-binding $\beta$ registration $^{24}$ when AAA1 is bound to ATP and AAA3 is in the posthydrolysis state ${ }^{42}$. To account for these findings, we have refined the dynein model we have recently described ${ }^{24}$ (Fig. 7, step 1 and step 2). After detachment from the MT (Fig. 7, step 3), the trailing head is displaced forward by the priming stroke of its linker (Fig. 7, step 4). The priming stroke can only occur when AAA4 is bound to ATP (or if it assumes the ADP.Pi transition state) to open the gate on AAA3 and when ATP is bound to AAA1. In addition, AAA3 must be in the ADP.Pi transition state or bound to ADP to open the gate on AAA1 ${ }^{42,56}$. Following ATP hydrolysis, the tethered head then binds to a new binding site on the MT (Fig. 7, step 5). MT binding via initial weak interactions causes the power stroke (Fig. 7, step 6). The docking of the linker $\mathrm{N}$-terminus to AAA5 in the post-powerstroke state then allows the transition into the strong MT-binding $\alpha$-registration, which is capable of bearing sufficient force.

In summary, our data suggest that dynein uses a "two-doored", double "key-lock" mechanism ${ }^{66}$ where the AAA4 and AAA3 $3^{42}$ nucleotide states control the effects of AAA1-ATP binding and hydrolysis on both dynein-MT binding and linker conformational changes. Multi-layered regulation by dynein's AAA+ domains underscores the complex interactions among AAA1, 3, and 4, dynein MTBDs, and external and intramolecular tension that collectively control dynein mechanochemistry. The results presented here will enable future studies on the role of AAA4 in dynein mechanochemistry. It is possible that AAA4 presents a locus for dynein regulation. For example, dynein's regulatory protein lissencephaly-1 (Lis1), which is a dimer, binds to two distinct sites on dynein's AAA + ATPase ring, a site that overlaps

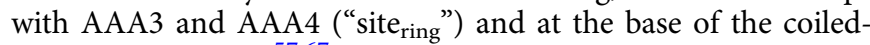
coil stalk ("site stalk") $^{57,67}$. It was shown that the interaction of Lis1 with site $_{\text {ring }}$ is responsible for an increased MT affinity and reduced velocity ${ }^{57,68}$, while the concurrent binding to both sites induces weak MT-binding and increased velocity ${ }^{57}$. It is therefore 


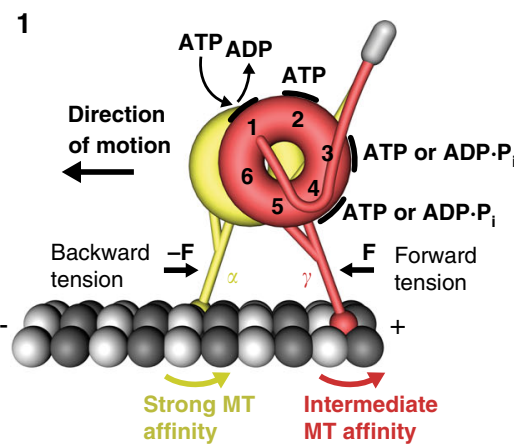

2

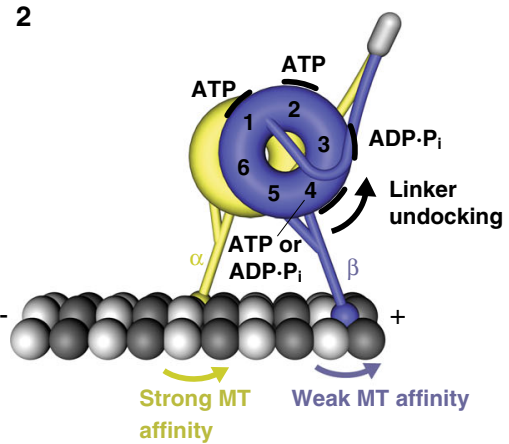

3

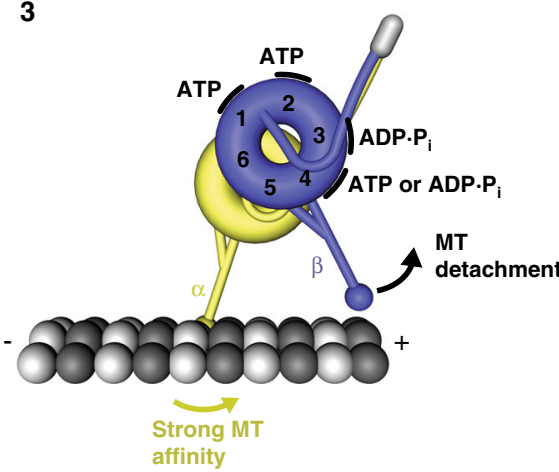

6

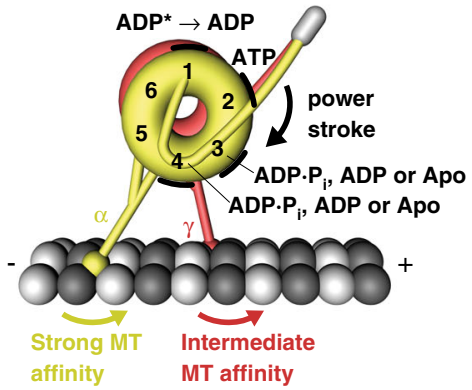

5

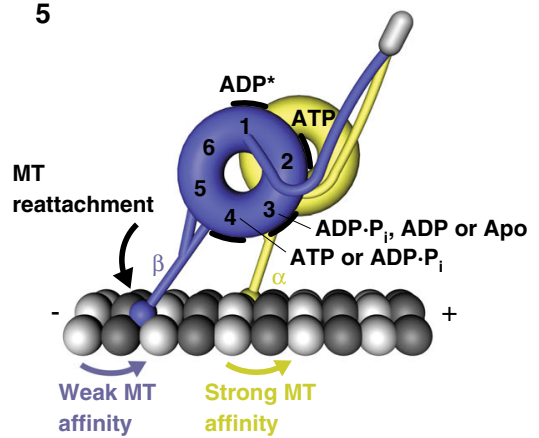

4

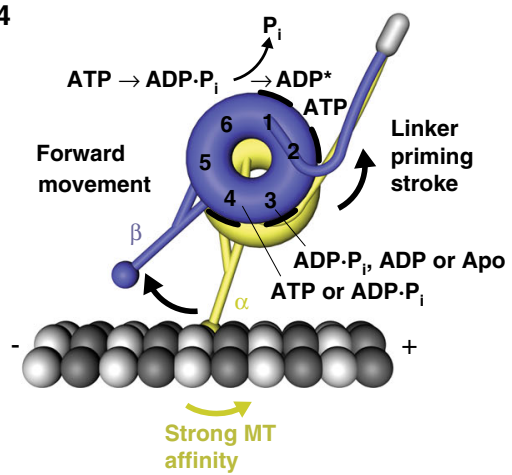

Fig. 7 Model for the mechanochemical cycle of dynein. Following ADP release from AAA1, ATP binding (step 1) causes the undocking of the linker from AAA5 and the subsequent transition from the $\gamma$ registry of the stalk helices with intermediate weak MT-affinity (red) to the weakly MT-binding $\beta$ registry (blue) only if AAA4 is bound to ATP or in the post-hydrolysis state (step 2). After the detachment of the rear head (step 3), which occurs when AAA3 is in the ADP • Pi transition state or bound to ADP, the 'recocking' of the linker (priming stroke) displaces the detached head forward to a new front MT-binding site while the MT-attached head bears the load (step 4). Following ATP hydrolysis and Pi release from AAA1 (step 4), rebinding to the MT in the weakly MT-binding $\beta$ registry (step 5) causes the transition from the 'high-energy ADP* state' to the 'low-energy ADP state', which generates a linker swing (powerstroke), resulting in the docking of the linker to AAA5 and the transition into the strong MT-binding $\alpha$ registry (yellow) of the stalk helices (step 6). A prerequisite for the strong binding state is that AAA3 is not bound to ADP, suggesting that AAA3 is still in the ADP • Pi state or nucleotide free. The MT minus-end-directed linker swing generates the forward movement of dynein's center of mass and the attached load.

possible that the reduction in dynein velocity as a result of the

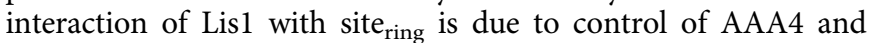
AAA3 function (as preventing ATP hydrolysis by AAA3 or AAA4 reduces dynein velocity, Fig. 2). Future studies will be necessary to determine whether Lis1 affects dynein motion by controlling the gating functions of AAA3 and/or AAA4. The observation that the binding of Lis1 to site stalk $_{\text {depends on the }}$ nucleotide state of AAA3 supports such a possibility ${ }^{57}$.

\section{Methods}

Generation of yeast strains. Mutant yeast stains (listed in Supplementary Table 1) were engineered as described previously ${ }^{24}$. Briefly, the PrimerQuest tool from IDT (Integrated DNA Technologies) was used to design PCR primers (see Supplementary Table 2 for the list of primers used), and DNA fragments were generated using KOD Hot Start DNA polymerase (EMD Millipore). Yeast transformation was performed using the Frozen-EZ Yeast Transformation II Kit (Zymo Research), followed by a two-step selection method using either synthetic media with uracil-dropout amino acid mix (SC/URA) or 5-fluorouracil (5-FOA) as the selective agents. All newly engineered and mutated yeast strains were confirmed by PCR and sequencing.

Yeast growth and protein expression. Full-length homodimeric yeast dynein, GFP-Dyn $1_{471 \mathrm{kDa}}$, and mutants were generated and purified as described recently ${ }^{69}$ Yeast cultures and protein production of the single-headed dynein, GFP-Dyn $1_{331}$ $\mathrm{kDa}$, and its mutants was done as described previously ${ }^{24}$ with the modifications described below. The parent strain used in this work was VY137, which expresses a tail-truncated minimal S. cerevisiae MD containing the linker, AAA+ ring, stalk, buttress, and MTBD. This construct retains its motor activities ${ }^{42,52,56}$ and is equivalent to the Dictyostelium discoideum MD used in key biochemical studies $^{19,22,37,39,70-72}$. The genotype was pGal:ZZ:Tev:GFP:HA:D6 MATa; his311,15; ura3-1; leu2-3,112; ade2-1; trp1-1; pep4A:HIS5; prb1 $\Delta$. Mutants were expressed behind the inducible galactose promotor $(p G A L)$. Yeast cells were grown in $5 \mathrm{~mL}$ of $2 \times$ YPD medium $(20 \mathrm{~g} / \mathrm{L}$ yeast extract, $40 \mathrm{~g} / \mathrm{L}$ peptone, $4 \%$ [w/v] dextrose) overnight, then transferred to $50 \mathrm{~mL}$ of YPR solution $(2 \%$ [w/v] raffinose $)$ and inoculated for $8 \mathrm{~h}$. For single-headed dynein, expression was induced by growing the cells in $2 \times$ YPG medium ( $4 \%$ [w/v] galactose) to a final $\mathrm{OD}_{600}$ between 
1.5 and $2.5(\sim 16 \mathrm{~h})$. After harvesting by centrifugation at $500 \times g$ for $5 \mathrm{~min}$, the pellets were resuspended in 0.2 volumes of $\mathrm{ddH}_{2} \mathrm{O}$ and flash-frozen in liquid nitrogen as small droplets. The cell pellets were stored at $-80^{\circ} \mathrm{C}$ until further use.

Yeast dynein purification. To purify the dynein, the frozen cell pellet droplets were pulverized using a kitchen coffee grinder that had been pre-chilled with liquid nitrogen. Next, 0.25 volumes of $4 \times$ lysis buffer $(1 \times$ lysis buffer: $30 \mathrm{mM}$ HEPES, $50 \mathrm{mM} \mathrm{KAc}, 2 \mathrm{mM} \mathrm{Mg}(\mathrm{Ac})_{2}, 1 \mathrm{mM}$ EGTA, $10 \%$ glycerol, $1 \mathrm{mM}$ DTT, $0.1 \mathrm{mM}$ Mg-ATP, $0.5 \mathrm{mM}$ Pefabloc, $10 \mathrm{ng} / \mathrm{mL}$ Leupeptin, $10 \mathrm{ng} / \mathrm{mL}$ Pepstatin A, and $0.2 \%$ $\mathrm{v} / \mathrm{v}$ Triton X-100, $\mathrm{pH} 7.2$ ) were added to reach a final $1 \times$ lysis buffer concentration. The cell lysate was cleared via ultracentrifugation at $80,000 \times g$ for $10 \mathrm{~min}$ at $4{ }^{\circ} \mathrm{C}$. Then, $0.25 \mathrm{~mL}$ of IgG sepharose 6 fast flow beads (GE Healthcare) were added to the supernatant and incubated for $1 \mathrm{~h}$ at $4{ }^{\circ} \mathrm{C}$ while rotating. The dynein-bound IgG beads were then washed with $\sim 10$ bead volumes of $1 \times$ lysis buffer, followed by a wash with $10 \mathrm{~mL}$ of $1 \times \mathrm{TEV}$ protease cleavage buffer $(30 \mathrm{mM}$ HEPES, $150 \mathrm{mM}$ KAc, $2 \mathrm{mM} \mathrm{Mg}(\mathrm{Ac})_{2}, 1 \mathrm{mM}$ EGTA, $10 \%$ glycerol, $0.1 \mathrm{mM}$ Mg-ATP, $0.5 \mathrm{mM}$ Pefabloc, and $0.1 \% \mathrm{v} / \mathrm{v}$ Triton X-100, $\mathrm{pH} 7.2$ ). The beads were then resuspended in an equal volume of cleavage buffer and $2 \% \mathrm{v} / \mathrm{v}$ of AcTev protease (ThermoFisher Scientific) was added. The mixture was nutated at $4{ }^{\circ} \mathrm{C}$ for $2 \mathrm{~h}$ to cleave dynein from the IgG beads. The mixture was centrifuged at $1000 \times g$ for $1 \mathrm{~min}$ at $4^{\circ} \mathrm{C}$, and the dynein-containing supernatant was then collected, flash-frozen with liquid nitrogen, and stored at $-80^{\circ} \mathrm{C}$.

MT binding and release purification of dynein. MT binding and release purification was performed to further purify the dynein and to remove any inactive motors and aggregates. To $50 \mu \mathrm{L}$ of TEV-released dynein, $0.1 \mu \mathrm{L}$ of $10 \mathrm{mM}$ paclitaxel and $5 \mu \mathrm{L}$ of $5 \mathrm{mg} / \mathrm{mL}$ paclitaxel-stabilized MTs were added. This solution was then layered onto a $100 \mu \mathrm{L}$ sucrose cushion $(30 \mathrm{mM}$ HEPES, $200 \mathrm{mM} \mathrm{KCl}, 2 \mathrm{mM} \mathrm{MgCl}_{2}$, pH 7.4, $10 \%$ v/v glycerol, $25 \% \mathrm{w} / \mathrm{v}$ sucrose, $1 \mathrm{mM}$ DTT, and $20 \mu \mathrm{M}$ paclitaxel) and centrifuged at $25^{\circ} \mathrm{C}$ for $10 \mathrm{~min}$ at $50,000 \times g$ After removing the supernatant, the pellet was carefully rinsed with $60 \mu \mathrm{L}$ of wash buffer ( $30 \mathrm{mM}$ HEPES, $150 \mathrm{mM} \mathrm{KCl}, 2 \mathrm{mM} \mathrm{MgCl}, 10 \%$ glycerol, $1 \mathrm{mM}$ EGTA, $1 \mathrm{mM}$ DTT, and $20 \mu \mathrm{M}$ paclitaxel, $\mathrm{pH}$ 7.2) and resuspended in $52 \mu \mathrm{L}$ of wash buffer with $6 \mathrm{mM} \mathrm{Mg-ATP}$. After incubating for $2 \mathrm{~min}$ at room temperature, the solution was centrifuged for $5 \mathrm{~min}$ at $50,000 \times g$. The MT suspension was then collected, aliquoted in $2 \mu \mathrm{L}$ volumes, and flash-frozen in liquid nitrogen before storing at $-80^{\circ} \mathrm{C}$.

Flow chamber preparation and MT immobilization. Flow chambers were prepared as described previously ${ }^{73}$. Briefly, $18 \times 18 \times 0.17-\mathrm{mm}$ coverslips (Zeiss) were placed in a porcelain coverslip rack using forceps and submerged in $\mathrm{HNO}_{3}$ $(25 \% \mathrm{v} / \mathrm{v})$ for $15 \mathrm{~min}$ followed by rinsing with $\mathrm{ddH}_{2} \mathrm{O}$. Then, the rack with the coverslips was placed in $\mathrm{NaOH}$ for 2 to $5 \mathrm{~min}$, followed by rinsing extensively with $\mathrm{ddH}_{2} \mathrm{O}$. After drying on a heating block for $30 \mathrm{~min}$, the coverslips were store in a vacuum desiccator. The flow chambers were assembled as described ${ }^{8}$. To immobilize MTs on the coverslip surface, $10 \mu \mathrm{L}$ of $5 \mathrm{mg} / \mathrm{mL}$ biotinylated $\alpha$ casein was flowed into the slide chamber and incubated for $10 \mathrm{~min}$. The chamber was then washed three times with $20 \mu \mathrm{L}$ of blocking buffer ("BB", $80 \mathrm{mM}$ PIPES, $2 \mathrm{mM} \mathrm{MgCl}, 1 \mathrm{mM}$ EGTA, $1 \%$ Pluronic F-127, $1 \mathrm{mg} / \mathrm{mL} \alpha$-casein, and $20 \mu \mathrm{M}$ Taxol) and incubated for $1 \mathrm{~h}$ to fully block the glass surface. The slide chamber was then washed twice with $20 \mu \mathrm{L}$ of $\mathrm{BB}$, and the solution inside the chamber was completely removed. Subsequently, $12 \mu \mathrm{L}$ of $1 \mathrm{mg} / \mathrm{mL}$ streptavidin was flowed into the chamber and incubated for $10 \mathrm{~min}$. The chamber was then washed three times with $20 \mu \mathrm{L}$ of BB. Finally, $20 \mu \mathrm{L}$ of BB that contained the suspended MTs and $10 \mu \mathrm{M}$ paclitaxel was flowed into the chamber and immediately washed with $40 \mu \mathrm{L}$ of BB. Polarity-marked MTs were prepared as described previously ${ }^{24}$.

Single-molecule motility assay. To dimerize the single-headed dynein constructs, Cy3-labeled anti-GFP antibodies were used as previously described ${ }^{24}$. Dynein constructs were diluted to the appropriate concentration using HME30 $(30 \mathrm{mM}$ HEPES, $2 \mathrm{mM} \mathrm{MgCl}_{2}$, and $1 \mathrm{mM}$ EGTA) and incubated on ice with Cy3-labeled, anti-GFP antibodies for $10 \mathrm{~min}$. A final motility buffer (MB) containing $1 \mathrm{mM}$ ATP, $1 \mathrm{mg} / \mathrm{mL}$ a-casein, $20 \mu \mathrm{M}$ Taxol, $75 \mathrm{mM} \mathrm{KCl}, 2 \mathrm{mM}$ Trolox, Gloxy, $1 \mathrm{mM}$ TCEP, and the motor-antibody mixture was flowed into the chamber. MTs and dynein were visualized with a custom-built TIRF microscope equipped with an Andor iXon Ultra EMCCD. MTs were first imaged by taking a single-frame snapshot. Dynein was then imaged with an acquisition time of $500 \mathrm{~ms}$ and a total 500 frames were acquired for each movie. Finally, the MTs were imaged again by taking a snapshot to check for stage drift. Movies with significant drift were not analyzed. Each sample was imaged no longer than $15 \mathrm{~min}$. The kymographs were generated using Fiji and the data were analyzed using a custom written MATLAB program.

Stall-force measurements. Stall-force measurements were performed using a custom-built force-fluorescence microscope as described previously 27,50 . Briefly, TMR-labeled MTs were immobilized to glass coverslip as described above. Fulllength yeast dynein was diluted to the appropriate concentrations using trapping buffer ( $30 \mathrm{mM}$ HEPES, $2 \mathrm{mM} \mathrm{MgAc}_{2}, 1 \mathrm{mM}$ EGTA, $1 \mathrm{mM}$ TCEP, $50 \mathrm{mM}$ KAc
$0.75 \mathrm{mg} / \mathrm{mL}$ a-casein, $20 \mu \mathrm{M}$ paclitaxel, $20 \mathrm{mM}$ glucose, and $2 \mathrm{mM}$ Trolox, $\mathrm{pH} 7.2$ ) and incubated for $10 \mathrm{~min}$ on ice with anti-GFP antibody-coated $\sim 1-\mu \mathrm{m}$ diameter beads $(980 \mathrm{~nm}$, carboxyl-modified polystyrene microspheres, Bangs Laboratories) The mixture was then supplemented with $1 \mathrm{mM}$ ATP and Gloxy (a glucose oxidase and catalase-based oxygen scavenging system), and flown into the slide chamber. Trapping assays were performed at $25^{\circ} \mathrm{C}$ as previously described at trap stiffnesses of $0.03-0.06 \mathrm{pN} / \mathrm{nm}^{59}$.

MT-gliding assay. The flow chamber was assembled as described above. To immobilize dynein on the coverslip surface, $10 \mu \mathrm{L}$ of $0.4 \mathrm{mg} / \mathrm{ml}$ rabbit monoclonal anti-GFP antibody was flown into the slide chamber and incubated for $10 \mathrm{~min}$. The chamber was then washed twice with $20 \mu \mathrm{L}$ blocking buffer ("BB", $80 \mathrm{mM}$ PIPES, $2 \mathrm{mM} \mathrm{MgCl} 2,1 \mathrm{mM}$ EGTA, $1 \%$ Pluronic F-127, $1 \mathrm{mg} / \mathrm{mL} \alpha-$ casein) and incubated for $10 \mathrm{~min}$ to block the glass surface. The slide chamber was then washed twice with motility buffer ("MB", $30 \mathrm{mM}$ HEPES, $2 \mathrm{mM} \mathrm{MgCl}$, $1 \mathrm{mM}$ EGTA, $1 \mathrm{mg} / \mathrm{mL} \alpha$-casein, $50 \mathrm{mM} \mathrm{KCl}, 1 \mathrm{mM}$ TCEP) before $10 \mu \mathrm{L}$ dynein solution (diluted to an appropriate concentration in MB) was flown into the slide chamber and incubated for $2 \mathrm{~min}$. The flow chamber was then washed twice with $20 \mu \mathrm{L} \mathrm{MB}$ to remove excess dynein. A final $10 \mu \mathrm{L}$ motility buffer containing TMR-labeled MTs and suppled with $1 \mathrm{mM} \mathrm{ATP}, 20 \mu \mathrm{M}$ Taxol, $2 \mathrm{mM}$ Trolox and Gloxy was flown into the chamber. Microtubules were visualized with a custombuilt TIRF microscope described above and imaged with an acquisition time of $250 \mathrm{~ms}$ for a total 1200 frames per movie. The kymographs were generated using Fiji.

Unbinding-force assay. Unbinding-force measurements were performed as described previously 24,42 . Briefly, polarity-marked MTs were attached to the flow chamber coverslip as described above. Yeast dynein was diluted to the appropriate concentration using trapping buffer (30 mM HEPES, $2 \mathrm{mM} \mathrm{MgAc}_{2}, 1 \mathrm{mM}$ EGTA, $20 \mu \mathrm{M}$ paclitaxel, $20 \mathrm{mM}$ glucose, and $2 \mathrm{mM}$ Trolox, $\mathrm{pH}$ 7.2) and incubated on ice with anti-GFP antibody Fab fragment-coated $\sim 1$ - $\mu$ m diameter beads ( $980 \mathrm{~nm}$, carboxyl-modified polystyrene microspheres, Bangs Laboratories) for $10 \mathrm{~min}$. To remove free unbound motors, the beads were centrifuged at $4{ }^{\circ} \mathrm{C}$ for $2 \mathrm{~min}$ at $3000 \times \mathrm{g}$, followed by supernatant removal. The beads were then resuspended with $40 \mu \mathrm{L}$ trapping buffer containing $0.75 \mathrm{mg} / \mathrm{mL}$ a-casein, Gloxy (a glucose oxidase and catalase-based oxygen scavenging system) and 6.6 units/ $\mathrm{mL}$ Apyrase (to remove residual ATP for the apo state experiments) or $1 \mathrm{mM}$ ATP. A bead bearing a dynein MD was then held in an optical trap as the nanopositioning stage swept back and forth parallel to a surface-bound MT. The stage speed was adjusted to produce an apparent loading rate of $5.6 \mathrm{pN} / \mathrm{s}$ (the true loading rate depends on the compliance of the motor and is smaller than the apparent rate $\left.^{24}\right)$.

Analysis of data generated by the constant-pulling assay. As we showed previously ${ }^{42}$, the largest forces in our constant-pulling unbinding experiments (Fig. 3a) usually occur when a bead rebinds to the MT before returning to the trap center. We call these secondary binding/unbinding events. For primary events, zero force was applied to the MD immediately after MT binding $\left(F_{\text {start }}=0\right)$, but for secondary events, $F_{\text {start }}>0$. Because the history of force applied to the bond depends on $F_{\text {start }}$, we only focused on primary events (Fig. 3a, right). Measurements from multiple beads and experiments under the same conditions were pooled together and used to generate unbinding-force histograms with 1-pN bins (as our force detection limit is $\sim 0.3 \mathrm{pN}$, we only plotted the force-dependent unbinding rates for forces above $0.5 \mathrm{pN}$ ). To compare the unbinding-force distributions and the derived force-dependent unbinding rates for both loading directions, we plotted the data as a function of the absolute force values. Normalized histograms that approximated the probability density functions for unbinding at a given force were then calculated. The value of each bin was divided by $N$, the total number of unbinding-force measurements. Because the unbinding-force distributions were not normally distributed, we estimated the sampling error by bootstrapping. For each histogram, 95\% confidence intervals (CIs) for the mean statistic were calculated using the MATLAB bootci() function as described previously ${ }^{42}$. To estimate $p$-values when comparing means of different distributions, we first created a dataset representing the sampling distribution of the mean for each original dataset. First, we bootstrapped $10^{5}$ means with the MATLAB function bootstrp(). We then subtracted these means pairwise to create a dataset representing the sampling distribution of the difference of the means. From each measurement in this dataset, we subtracted the mean difference of the means, which shifted the mean of the distribution to zero. This transformation was consistent with the null hypothesis of no difference between the original unbinding-force distribution means. The $p$-value $\left(p_{\mathrm{m}}\right)$ was then calculated as the proportion of the bootstrapped mean differences that were greater than or equal to the difference observed between the means of the original datasets (two-tailed test). Similar to our recent work $^{24}$, we considered the compliance of the dynein motor and bead linkage, which resulted in a forcedependent loading rate. Uncertainty arises in the calculated unbinding rates as a function of force due to limited statistics for larger forces. To reduce this uncertainty, we used a kernel density estimator to describe the probability density functions of the measured unbinding forces before transforming them into forcedependent unbinding rates ${ }^{61}$. 
Reporting summary. Further information on research design is available in the Nature Research Reporting Summary linked to this article.

\section{Data availability}

Data supporting the findings of this manuscript are available from the corresponding author upon reasonable request. A reporting summary for this Article is available as a Supplementary Information file.

Received: 20 May 2020; Accepted: 19 October 2020;

Published online: 23 November 2020

\section{References}

1. Roberts, A. J., Kon, T., Knight, P. J., Sutoh, K. \& Burgess, S. A. Functions and mechanics of dynein motor proteins. Nat. Rev. Mol. Cell Biol. 14, 713-726 (2013).

2. Olenick, M. A. \& Holzbaur, E. L. F. Dynein activators and adaptors at a glance. J. Cell Sci. 132, jcs227132 (2019).

3. Kardon, J. R. \& Vale, R. D. Regulators of the cytoplasmic dynein motor. Nat. Rev. Mol. Cell Biol. 10, 854-865 (2009).

4. Reck-Peterson, S. L., Redwine, W. B., Vale, R. D. \& Carter, A. P. The cytoplasmic dynein transport machinery and its many cargoes. Nat. Rev. Mol. Cell Biol. 19, 382-398 (2018).

5. Peeters, K. et al. Novel mutations in the DYNC1H1 tail domain refine the genetic and clinical spectrum of dyneinopathies. Hum. Mutat. 36, 287-291 (2015).

6. Gelineau-Morel, R. et al. Congenital cataracts and gut dysmotility in a DYNC1H1 dyneinopathy patient. Genes 7, 85-92 (2016).

7. Poirier, K. et al. Mutations in TUBG1, DYNC1H1, KIF5C and KIF2A cause malformations of cortical development and microcephaly. Nat. Genet. 45, 639-647 (2013).

8. Willemsen, M. H. et al. Mutations in DYNC1H1 cause severe intellectual disability with neuronal migration defects. J. Med. Genet. 49, 179-183 (2012).

9. Fiorillo, C. et al. Novel dynein DYNC1H1 neck and motor domain mutations link distal spinal muscular atrophy and abnormal cortical development. Hum. Mutat. 35, 298-302 (2014).

10. Harms, M. B. et al. Mutations in the tail domain of DYNC1H1 cause dominant spinal muscular atrophy. Neurology 78, 1714-1720 (2012).

11. Neveling, K. et al. Mutations in BICD2, which encodes a golgin and important motor adaptor, cause congenital autosomal-dominant spinal muscular atrophy. Am. J. Hum. Genet. 92, 946-954 (2013).

12. Niu, Q., Wang, X., Shi, M. \& Jin, Q. A novel DYNC1H1 mutation causing spinal muscular atrophy with lower extremity predominance. Neurol. Genet. 1, e20 (2015)

13. Chan, S. H. S. et al. A recurrent de novo DYNC1H1 tail domain mutation causes spinal muscular atrophy with lower extremity predominance, learning difficulties and mild brain abnormality. Neuromuscul. Disord. 28, 750-756 (2018).

14. Weedon, M. N. et al. Exome sequencing identifies a DYNC1H1 mutation in a large pedigree with dominant axonal Charcot-Marie-Tooth disease. Am. J. Hum. Genet. 89, 308-312 (2011).

15. Strickland, A. V. et al. Mutation screen reveals novel variants and expands the phenotypes associated with DYNC1H1. J. Neurol. 262, 2124-2134 (2015)

16. Ding, D. et al. Identification of a de novo DYNC1H1 mutation via WES according to published guidelines. Sci. Rep. 6, 20423 (2016).

17. Carter, A. P., Cho, C., Jin, L. \& Vale, R. D. Crystal structure of the dynein motor domain. Science 331, 1159-1165 (2011).

18. Kon, T., Sutoh, K. \& Kurisu, G. X-ray structure of a functional full-length dynein motor domain. Nat. Struct. Mol. Biol. 18, 638-642 (2011).

19. Roberts, A. J. et al. AAA + Ring and linker swing mechanism in the dynein motor. Cell 136, 485-495 (2009).

20. Roberts, A. J. et al. ATP-driven remodeling of the linker domain in the dynein motor. Structure 20, 1670-1680 (2012).

21. Kon, T. et al. The $2.8 \AA$ crystal structure of the dynein motor domain. Nature 484, 345-350 (2012).

22. Kon, T., Mogami, T., Ohkura, R., Nishiura, M. \& Sutoh, K. ATP hydrolysis cycle-dependent tail motions in cytoplasmic dynein. Nat. Struct. Mol. Biol. 12, 513-519 (2005).

23. Burgess, S. A., Walker, M. L., Sakakibara, H., Knight, P. J. \& Oiwa, K. Dynein structure and power stroke. Nature 421, 715-718 (2003).

24. Rao, L., Berger, F., Nicholas, M. P. \& Gennerich, A. Molecular mechanism of cytoplasmic dynein tension sensing. Nat. Commun. 10, 3332 (2019).

25. Gennerich, A. \& Vale, R. D. Walking the walk: how kinesin and dynein coordinate their steps. Curr. Opin. Cell Biol. 21, 59-67 (2009).

26. Trokter, M., Mücke, N. \& Surrey, T. Reconstitution of the human cytoplasmic dynein complex. Proc. Natl Acad. Sci. USA 109, 20895-20900 (2012).
27. Nicholas, M. P. et al. Control of cytoplasmic dynein force production and processivity by its C-terminal domain. Nat. Commun. 6, 6206 (2015).

28. McKenney, R. J., Huynh, W., Tanenbaum, M. E., Bhabha, G. \& Vale, R. D. Activation of cytoplasmic dynein motility by dynactin-cargo adapter complexes. Science 345, 337-341 (2014)

29. Schlager, M. A., Hoang, H. T., Urnavicius, L., Bullock, S. L. \& Carter, A. P. In vitro reconstitution of a highly processive recombinant human dynein complex. EMBO J. 33, 1855-1868 (2014).

30. Torisawa, T. et al. Autoinhibition and cooperative activation mechanisms of cytoplasmic dynein. Nat. Cell Biol. 16, 1118-1124 (2014).

31. Toropova, K., Mladenov, M. \& Roberts, A. J. Intraflagellar transport dynein is autoinhibited by trapping of its mechanical and track-binding elements. Nat. Struct. Mol. Biol. 24, 461-468 (2017).

32. Zhang, $\mathrm{K}$. et al. Cryo-EM reveals how human cytoplasmic dynein is autoinhibited and activated. Cell 169, 1303-1314 (2017).

33. Chowdhury, S., Ketcham, S. A., Schroer, T. A. \& Lander, G. C. Structural organization of the dynein-dynactin complex bound to microtubules. Nat. Struct. Mol. Biol. 22, 345-347 (2015).

34. Belyy, V. et al. The mammalian dynein-dynactin complex is a strong opponent to kinesin in a tug-of-war competition. Nat. Cell Biol. 18, 1018-1024 (2016).

35. Grotjahn, D. A. et al. Cryo-electron tomography reveals that dynactin recruits a team of dyneins for processive motility. Nat. Struct. Mol. Biol. 25, 203-207 (2018).

36. Urnavicius, L. et al. Cryo-EM shows how dynactin recruits two dyneins for faster movement. Nature 554, 202-206 (2018).

37. Kon, T. et al. Helix sliding in the stalk coiled coil of dynein couples ATPase and microtubule binding. Nat. Struct. Mol. Biol. 16, 325-333 (2009).

38. Gibbons, I. R. et al. The affinity of the dynein microtubule-binding domain is modulated by the conformation of its coiled-coil stalk. J. Biol. Chem. $\mathbf{2 8 0}$ 23960-23965 (2005).

39. Imamula, K., Kon, T., Ohkura, R. \& Sutoh, K. The coordination of cyclic microtubule association/dissociation and tail swing of cytoplasmic dynein Proc. Natl Acad. Sci. USA 104, 16134-16139 (2007).

40. Holzbaur, E. L. \& Johnson, K. A. Microtubules accelerate ADP release by dynein. Biochemistry 28, 7010-7016 (1989).

41. Porter, M. E. \& Johnson, K. A. Transient state kinetic analysis of the ATPinduced dissociation of the dynein-microtubule complex. J. Biol. Chem. 258, 6582-6587 (1983)

42. Nicholas, M. P. et al. Cytoplasmic dynein regulates its attachment to microtubules via nucleotide state-switched mechanosensing at multiple AAA domains. Proc. Natl Acad. Sci. USA 112, 6371-6376 (2015).

43. DeWitt, M. A., Cypranowska, C. A., Cleary, F. B., Belyy, V. \& Yildiz, A. The AAA3 domain of cytoplasmic dynein acts as a switch to facilitate microtubule release. Nat. Struct. Mol. Biol. 22, 73-80 (2015).

44. Carter, A. P. et al. Structure and functional role of dynein's microtubulebinding domain. Science 322, 1691-1695 (2008).

45. McNaughton, L., Tikhonenko, I., Banavali, N. K., LeMaster, D. M. \& Koonce M. P. A low affinity ground state conformation for the Dynein microtubule binding domain. J. Biol. Chem. 285, 15994-16002 (2010).

46. Uchimura, S. et al. A flipped ion pair at the dynein-microtubule interface is critical for dynein motility and ATPase activation. J. Cell Biol. 208, 211-222 (2015).

47. Cho, C., Reck-Peterson, S. L. \& Vale, R. D. Regulatory ATPase sites of cytoplasmic dynein affect processivity and force generation. J. Biol. Chem. 283 25839-25845 (2008)

48. Kon, T., Nishiura, M., Ohkura, R., Toyoshima, Y. Y. \& Sutoh, K. Distinct functions of nucleotide-binding/hydrolysis sites in the four AAA modules of cytoplasmic dynein. Biochemistry 43, 11266-11274 (2004).

49. Gennerich, A. Optical Tweezers: Methods and Protocols (Humana Press, New York, 2017).

50. Nicholas, M. P., Rao, L. \& Gennerich, A. An improved optical tweezers assay for measuring the force generation of single Kinesin molecules. Methods Mol. Biol. 1136, 171-246 (2014).

51. Rao, L. et al. The yeast dynein Dyn2-Pac11 complex is a dynein dimerization/ processivity factor: structural and single-molecule characterization. Mol. Biol. Cell 24, 2362-2377 (2013)

52. Reck-Peterson, S. L. et al. Single-molecule analysis of dynein processivity and stepping behavior. Cell 126, 335-348 (2006).

53. Reck-Peterson, S. L., Vale, R. D. \& Gennerich, A. Motile properties of cytoplasmic dynein. In Handbook of Dynein (ed. Amos, K.H.a.L.) 145-172 (Pan Stanford Publishing, Singapore, 2012).

54. Neuwald, A. F., Aravind, L., Spouge, J. L. \& Koonin, E. V. AAA+: a class of chaperone-like ATPases associated with the assembly, operation, and disassembly of protein complexes. Genome Res. 9, 27-43 (1999).

55. Ogura, T. \& Wilkinson, A. J. AAA+ superfamily ATPases: common structurediverse function. Genes Cells 6, 575-597 (2001).

56. Cleary, F. B. et al. Tension on the linker gates the ATP-dependent release of dynein from microtubules. Nat. Commun. 5, 4587 (2014). 
57. DeSantis, M. E. et al. Lis1 has two opposing modes of regulating cytoplasmic dynein. Cell 170, 1197-1208 e12 (2017).

58. Gennerich, A. \& Reck-Peterson, S. L. Probing the force generation and stepping behavior of cytoplasmic dynein. Methods Mol. Biol. 783, 63-80 (2011).

59. Brenner, S., Berger, F., Rao, L., Nicholas, M. P. \& Gennerich, A. Force production of human cytoplasmic dynein is limited by its processivity. Sci. Adv. 6, eaaz4295 (2020).

60. Schmidt, H., Zalyte, R., Urnavicius, L. \& Carter, A. P. Structure of human cytoplasmic dynein-2 primed for its power stroke. Nature 518, 435-438 (2015).

61. Dudko, O. K., Hummer, G. \& Szabo, A. Theory, analysis, and interpretation of single-molecule force spectroscopy experiments. Proc. Natl Acad. Sci. USA 105, 15755-15760 (2008).

62. Schmidt, H., Gleave, E. S. \& Carter, A. P. Insights into dynein motor domain function from a 3.3-Å crystal structure. Nat. Struct. Mol. Biol. 19, 492-497 (2012).

63. Bhabha, G. et al. Allosteric communication in the dynein motor domain. Cell 159, 857-868 (2014).

64. Gennerich, A., Carter, A. P., Reck-Peterson, S. L. \& Vale, R. D. Force-induced bidirectional stepping of cytoplasmic dynein. Cell 131, 952-965 (2007).

65. Ezber, Y., Belyy, V., Can, S. \& Yildiz, A. Dynein harnesses active fluctuations of microtubules for faster movement. Nat. Phys. 16, 312-+ (2020).

66. Leysen, S., Vanderkelen, L., Weeks, S. D., Michiels, C. W. \& Strelkov, S. V. Structural basis of bacterial defense against g-type lysozyme-based innate immunity. Cell Mol. Life Sci. 70, 1113-1122 (2013).

67. Htet, Z. M. et al. LIS1 promotes the formation of activated cytoplasmic dynein-1 complexes. Nat. Cell Biol. 22, 518-525 (2020).

68. Huang, J., Roberts, A. J., Leschziner, A. E. \& Reck-Peterson, S. L. Lis1 acts as a "Clutch" between the ATPase and microtubule-binding domains of the dynein motor. Cell 150, 975-986 (2012).

69. Rao, L., Hulsemann, M. \& Gennerich, A. Combining structure-function and single-molecule studies on cytoplasmic dynein. Methods Mol. Biol. 1665, 53-89 (2018)

70. Mizuno, N., Narita, A., Kon, T., Sutoh, K. \& Kikkawa, M. Three-dimensional structure of cytoplasmic dynein bound to microtubules. Proc. Natl Acad. Sci. USA 104, 20832-20837 (2007).

71. Mogami, T., Kon, T., Ito, K. \& Sutoh, K. Kinetic characterization of tail swing steps in the ATPase cycle of Dictyostelium cytoplasmic dynein. J. Biol. Chem. 282, 21639-21644 (2007).

72. Shima, T., Kon, T., Imamula, K., Ohkura, R. \& Sutoh, K. Two modes of microtubule sliding driven by cytoplasmic dynein. Proc. Natl Acad. Sci. USA 103, 17736-17740 (2006).

73. Nicholas, M. P., Rao, L. \& Gennerich, A. Covalent immobilization of microtubules on glass surfaces for molecular motor force measurements and other single-molecule assays. Methods Mol. Biol. 1136, 137-169 (2014).

\section{Acknowledgements}

A.G. thanks Lisa Baker for helpful discussions and for help with the editing of the manuscript. All authors were supported by the National Institute of Health (NIH) grant R01GM098469. X.L. also received support from a Pilot Project Grant from the Rose F. Kennedy Intellectual and Developmental Disabilities Research Center at the Albert Einstein College of Medicine.

\section{Author contributions}

X.L., L.R., and A.G. designed the research. X.L. performed the research and engineered the mutant yeast strains. X.L. also produced and purified the proteins. X.L. and A.G. analyzed the data and wrote the paper.

\section{Competing interests}

The authors declare no competing interests.

\section{Additional information}

Supplementary information is available for this paper at https://doi.org/10.1038/s41467020-19477-3.

Correspondence and requests for materials should be addressed to A.G.

Peer review information Nature Communications thanks the anonymous reviewers for their contributions to the peer review of this work.

Reprints and permission information is available at http://www.nature.com/reprints

Publisher's note Springer Nature remains neutral with regard to jurisdictional claims in published maps and institutional affiliations.

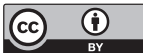

Open Access This article is licensed under a Creative Commons Attribution 4.0 International License, which permits use, sharing, adaptation, distribution and reproduction in any medium or format, as long as you give appropriate credit to the original author(s) and the source, provide a link to the Creative Commons license, and indicate if changes were made. The images or other third party material in this article are included in the article's Creative Commons license, unless indicated otherwise in a credit line to the material. If material is not included in the article's Creative Commons license and your intended use is not permitted by statutory regulation or exceeds the permitted use, you will need to obtain permission directly from the copyright holder. To view a copy of this license, visit http://creativecommons.org/ licenses/by/4.0/.

(C) The Author(s) 2020 